\title{
Synthesis of Discrete-Time Nonlinear Feedforward/Feedback Controllers
}

\author{
Masoud Soroush and Costas Kravaris \\ Dept. of Chemical Engineering, University of Michigan, Ann Arbor, MI 48109
}

\begin{abstract}
This work concerns the synthesis of discrete-time feedforward/feedback control systems for general nonlinear processes with stable zero dynamics. Depending on the process under consideration, the derived feedforward/feedback controllers can completely eliminate the effect of measurable disturbances and produce a prespecified linear response with respect to a reference input, or provide integral-square error optimal response to step changes in the disturbances and a prespecified linear response with respect to a reference input. In either case, the developed feedforward/ feedback controllers allow for the asymptotic rejection of unmeasurable disturbances. These controllers are derived within the globally linearizing control framework, first under full state information and then in the absence of state measurements. The internal stability of the closed-loop system is addressed. The derived controllers are interpreted from a model-predictive point of view, and their connections with the feedforward internal model control and the model algorithmic control are established. The theoretical results are illustrated through a continuous stirred-tank reactor example.
\end{abstract}

\section{Introduction}

In linear and nonlinear feedback controller design methods, the asymptotic rejection of disturbances is achieved by incorporating integral action in the controllers. In the case of measurable process disturbances, it is possible to take corrective actions before these disturbances upset the process, and therefore, reject them in a much more efficient way. The need for more efficient rejection of disturbances has motivated the development of feedforward/feedback control methods (Calvet and Arkun, 1988a,b; Daoutidis and Kravaris, 1989; Daoutidis et al., 1990; Garcia and Morari, 1985; Palmor and Powers, 1981). The original idea of feedforward control has been traced back to the 1920s, and feedforward control has been used widely in industry (Shinskey, 1988).

The inadequacy of linear controllers for nonlinear processes and the availability of new powerful mathematical tools have motivated an expanding research effort towards the development of nonlinear process control methods. So far, two major research directions have been pursued: the model predictive approach and the geometric approach.

Current address of M. Soroush: Dept. of Chemical Engineering, Drexel University, Philadelphia, PA 19104.
Model predictive control (MPC) methods have been implemented in industry since the late 1970 s. The popularity of MPC techniques began with the successful application of the two MPC methods, model algorithmic control (MAC) (Richalet et al., 1978; Mehra and Rouhani, 1980) and Dynamic Matrix Control (Cutler and Ramaker, 1979; Prett and Gillette, 1979), to industrial processes. Using the internal model control (IMC) structure, Garcia and Morari (1982) showed that the MPC methods and theoretical $z$-domain methods are very closely related. The linear MPC results have also been extended for nonlinear systems (Biegler and Rawlings, 1991; Economou et al., 1986; Henson and Seborg, 1991; Hidalgo and Brosilow, 1990; Li et al., 1990; Pathwardhan et al., 1990; Sistu and Bequette, 1991).

Geometric process control methods have their roots in differential geometry. After about a decade of significant progress in understanding of the mathematical characteristics of nonlinear systems, many system theoretic properties of nonlinear systems are now well-understood (Isidori, 1989; Nijmeijer and van der Schaft, 1990) and this understanding has provided the theoretical foundations for nonlinear controller design. Within 
the differential geometric framework, the globally linearizing control (GLC) method (Kravaris and Chung, 1987; Soroush and Kravaris, 1992a) has been developed, and connections between the GLC and MPC have been established (Soroush and Kravaris, 1992a,b). In particular, it has been shown that MAC is a special case of the discrete-time GLC.

The objectives of this article are:

- To establish the physical importance of relative orders in discrete-time setting and the key role of the relative magnitudes of the relative orders (process time delays) in control system design and in the degree of achievable control quality.

- To develop a discrete-time feedforward/feedback control method for general nonlinear processes with stable zero dynamics.

- To establish concrete theoretical connections between the developed discrete-time feedforward/feedback controllers and the model predictive approaches and the feedforward IMC (Garcia and Morari, 1985). More precisely, we show that application of the discrete-time feedforward/error-feedback GLC to linear systems leads to the state-space realization of a feedforward MAC (a MAC which uses the measurements of measurable disturbances).

After formulating the feedforward/feedback control problem, the definition of relative orders (process time-delays) with respect to the manipulated and disturbance inputs will be given. Synthesis formulas for feedforward/state feedback laws will be derived. Next, the discrete-time feedforward GLC will be introduced. Two classes of feedforward/feedback controllers will be formulated depending on the availability of process state measurements. The general nonlinear results will be applied to linear systems with measurable disturbances, followed by a state-space reformulation of MAC. The linear controller (derived from the application of feedforward/error-feedback GLC to linear systems) and the state-space feedforward MAC will be shown to be equivalent. A nonlinear feedforward model algorithmic controller will then be developed and will be shown to be identical with the feedforward/error-feedback GLC. Finally, the feedforward/error-feedback GLC will be illustrated by a reactor example.

\section{Mathematical Preliminaries}

Consider single-input/single-output (SISO) systems with measurable disturbances described by a discrete-time statespace model of the form:

$$
\left\{\begin{aligned}
x(k+1) & =\Phi\left[x(k), u(k), d_{1}(k), \ldots, d_{p}(k)\right] \\
y(k) & =h[x(k)]
\end{aligned}\right.
$$

where $x=\left[x_{1} \ldots x_{n}\right]^{T} \in \mathbb{R}^{n}$ denotes the vector of state variables. $u \in \mathbb{R}$ and $y \in \mathbb{R}$ are the manipulated input and controlled output, respectively. $\left[d_{1} \ldots d_{p}\right]^{T} \in \mathbb{R}^{p}$ represents the vector of measurable process disturbances. Here all the variables are in deviation form. It is assumed that $x \in X \subset \mathbb{R}^{n}, d=\left[d_{1} \ldots\right.$ $\left.d_{p}\right]^{T} \in D \subset \mathbb{R}^{p}$ and $u \in U \subset \mathbb{R}$, where $X, D$ and $U$ are open connected sets that contain the nominal equilibrium point. $\Phi(x$, $u, d)$ is an analytic vector function on $X \times U \times D$ and $h(x)$ is an analytic scalar function on $X$.

The model of Eq. 1 can be viewed as the sampled-data representation of the continuous-time model:

$$
\left\{\begin{array}{l}
\dot{\bar{x}}(t)=f[\bar{x}(t)]+g[\bar{x}(t)] \bar{u}(t)+\sum_{j=1}^{p} \eta_{j}[\bar{x}(t)] \bar{d}_{j}(t) \\
\bar{y}(t)=h[\bar{x}(t)]
\end{array}\right.
$$

that is, $x(k+1)=\Phi\left[x(k), u(k), d_{1}(k), \ldots, d_{p}(k)\right]$ represents the solution at time $(k+1) \Delta t$ of the differential equations in Eq. 2 starting at time $k \Delta t$ in $\bar{x}(k \Delta t)=x(k)$ and with constant $\bar{u}(t)=u(k)$ and $\bar{d}_{j}(t)=d_{j}(k), j=1, \ldots, m$, where $\Delta t$ is the sampling period.

Unlike the continuous-time model of Eq. 2, the discretetime model of Eq. 1 is not affine in $d_{1}, \ldots, d_{p}$ and $u$. We consider the general discrete-time state-space model of Eq. 1 because the sampled-data representation of an affine continuous-time model is in general nonaffine, and even when the discrete-time model is affine, an input/output linearizing feedforward/state feedback of the process will be, in general, a nonaffine function of the external input. Note that if a continuous-time nonlinear model has deadtimes, its sampled-data representation will still be in the general form of Eq. 1.

\section{Problem Statement}

Consider the general class of the nonlinear processes described by the discrete-time model of Eq. 1 . In the case none of process disturbances can be measured, pure feedback controllers can be used to ensure the asymptotic rejection of the process disturbances. In linear and nonlinear feedback controller design methods (for example, IMC (Garcia and Morari, 1982, 1985) and GLC (Kravaris and Chung, 1987; Soroush and Kravaris, 1992a)), the asymptotic rejection of disturbances is achieved by incorporating integral action in the controllers. In the case of measurable process disturbances, it is possible to take corrective actions before these disturbances upset the process, and therefore, reject them in a much more efficient way. The need for more efficient rejection of disturbances has motivated the development of feedforward/feedback control methods (for example, Calvet and Arkun, 1988a,b; Daoutidis and Kravaris, 1989; Daoutidis et al., 1990; Garcia and Morari, 1985; Palmor and Powers, 1981).

In this article, our objective is to derive general synthesis formulas for discrete-time feedforward/feedback control systems, which can be used for all the nonlinear processes whose delay-free parts are minimum phase. In particular, we would like the derived feedforward/feedback control systems to be capable of:

- rejecting the effect of measurable disturbances as effectively as possible

- rejecting the effect of unmeasurable disturbances asymptotically

- inducing desirable closed-loop dynamics to the process under consideration

- tracking set-point changes without offset.

The key first step in our work is the development of static feedforward/state feedback laws of the form:

$$
u(k)=\Psi\left[x(k), d_{1}(k), \ldots, d_{p}(k), v(k)\right]
$$

with

$$
\frac{\partial}{\partial v} \Psi\left(x, d_{1}, \ldots, d_{p}, v\right) \not \equiv
$$




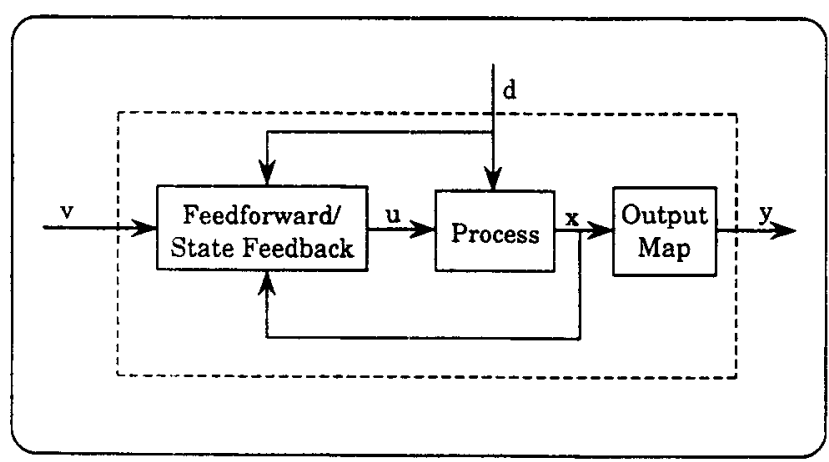

Figure 1. Feedforward/state feedback.

where $v \in \mathbb{R}$ is a reference input (see Figure 1). In addition, it is desirable to select static feedforward/state feedback laws of the form of Eq. 3, which can induce a desirable linear closedloop input/output behavior to the process under consideration. The linearity of the $v-y$ system facilitates greatly the analysis of the theoretical properties of the closed-loop system.

To asymptotically reject the unmeasurable disturbances and ensure offsetless tracking of set-point changes in the presence of modeling errors, we need a feedback controller with integral action around the $v-y$ loop. In the case that the $v-y$ system is linear, a linear error feedback controller with integral action around the $v-y$ system will induce linear input/output behavior to the overall system [linear set-point $\left(y_{s p}\right)$-output $(y)$ relation]. The linearity between the set-point $y_{s p}$ and the output $y$ facilitates the characterization and the study of the theoretical properties of the overall closed-loop system. The resulting control structure (feedforward/feedback law plus linear error feedback controller), which will be called the feedforward GLC structure, is shown in Figure 2. The control structure of Figure 2 is suitable when all the state variables $x$ can be measured online. When on-line measurements of states are not available and process is open-loop stable, an open-loop observer can be employed in conjunction with the feedforward/feedback law of Eq. 3 and the external linear error feedback controller, resulting in the control structure shown in Figure 3a. This control structure will be referred to as the feedforward/errorfeedback GLC structure.

\section{Relative Orders: Process Time Delays}

In analogy to the continuous-time feedforward/feedback problem (Daoutidis and Kravaris, 1989), a notion of relative order with respect to a disturbance input will be introduced. This relative order will play an instrumental role in the de-

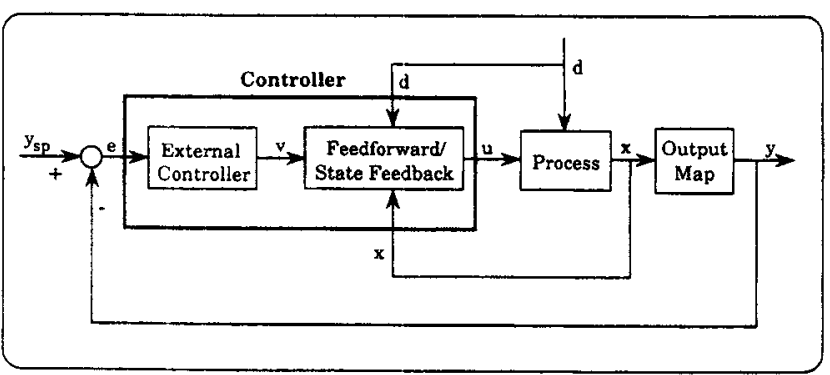

Figure 2. Feedforward GLC structure.

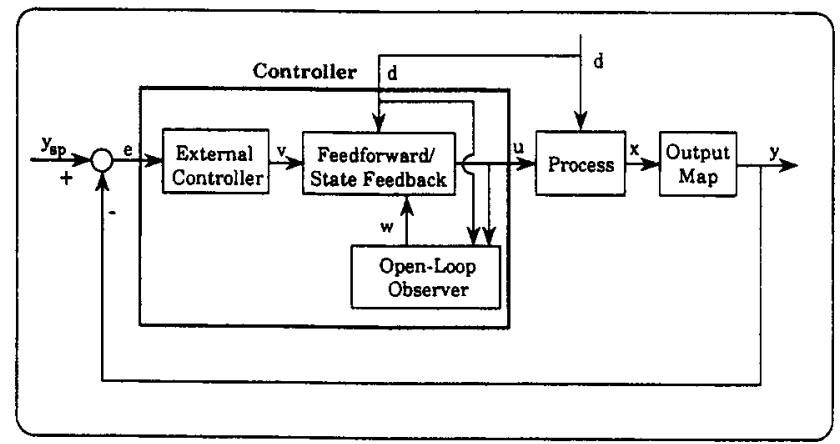

Figure 3a. Feedforward/error-feedback GLC structure.

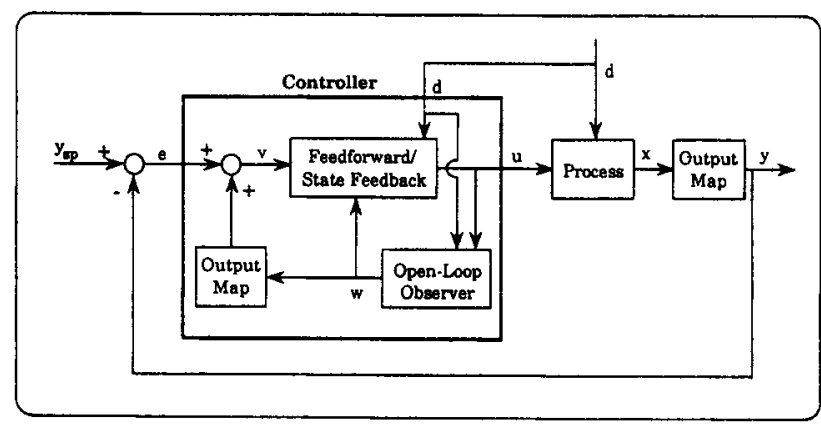

Figure 3b. Feedforward/error-feedback GLC (reducedorder) structure.

velopment of theory. The relative order with respect to the manipulated input will be defined similarly to the disturbancefree case (Nijmeijer and van der Schaft, 1990).

Definition 1. For a system of the form of Eq. 1, the relative order of the output $y$ with respect to the manipulated input $u$ is the smallest integer $r$ for which:

$$
\begin{aligned}
& {\left[\frac{\partial h(x)}{\partial x}\right]\left[\frac{\partial \Phi\left(x, u, d_{1}, \ldots, d_{p}\right)}{\partial x}\right]^{r-1} } \\
& \times\left[\frac{\partial \Phi\left(x, u, d_{1}, \ldots, d_{p}\right)}{\partial u}\right] \neq 0
\end{aligned}
$$

If such an integer does not exist, $r=\infty$. Equivalently, the relative order $r$ is the smallest integer for which:

$$
h \circ \frac{\Phi \circ \ldots \circ \Phi}{r \text { times }}
$$

depends on $u$. Therefore,

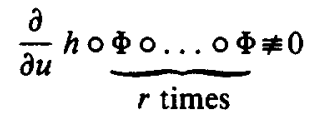

Here, $h \circ \Phi$ denotes the usual composition of functions $h$ and $\Phi: h \circ \Phi \Delta h\left[\Phi\left(x, u, d_{1}, \ldots, d_{p}\right)\right]$, and $\Phi \circ \Phi$ represents the usual composition of functions $\Phi$ and $\Phi$ in the variable $x$ : $\Phi \circ \Phi \triangleq$ $\Phi\left[\Phi\left(x, u, d_{1}, \ldots, d_{p}\right), u, d_{1}, \ldots, d_{p}\right]$.

Definition 2. For a system of the form of Eq. 1, the relative 
order of the output $y$ with respect to the disturbance $d_{j}$ is the smallest integer $\rho_{j}$ for which:

$$
\begin{aligned}
& {\left[\frac{\partial h(x)}{\partial x}\right]\left[\frac{\partial \Phi\left(x, u, d_{1}, \ldots, d_{p}\right)}{\partial x}\right]^{\rho_{j}-1} } \\
& \times\left[\frac{\partial \Phi\left(x, u, d_{1}, \ldots, d_{p}\right)}{\partial d_{j}}\right] \neq 0
\end{aligned}
$$

If such an integer does not exist, $\rho_{j}=\infty$. Equivalently, the relative order $\rho_{j}$ is the smallest integer for which:

$$
h \circ \frac{\Phi \circ \ldots \circ \Phi}{\rho_{j} \text { times }}
$$

$$
\frac{\partial}{\partial u} h \circ \frac{\Phi \circ \ldots \circ \Phi \neq 0}{r \text { times }}
$$

on $X \times U \times D$, that is, there is no singular point in $X \times U \times D$ at which the controller action $u(k)$ does not affect the predicted output $y(k+r)$.

\section{Effect of Static Feedforward/State Feedback on the Relative Orders}

Consider a nonlinear system of the form of Eq. 1 subject to a static feedforward/state feedback law of the form of Eq.

3. Then, the resulting closed-loop system is given by:

$$
\left\{\begin{aligned}
x(k+1) & =\Phi\left(x(k), \Psi\left(x(k), d_{1}(k), \ldots, d_{p}(k), v(k)\right), d_{1}(k), \ldots, d_{p}(k)\right) \\
y(k) & =h(x(k))
\end{aligned}\right.
$$

depends on $d_{j}$. Therefore:

$$
\frac{\partial}{\partial d_{j}} h \circ \Phi \circ \ldots \circ \Phi \neq 0
$$

In analogy to linear systems and along the lines of the nonlinear results given in (Nijmeijer and van der Schaft, 1990), one can show that the exact sampled-data representation of a deadtime-free SISO continuous-time system of the form of Eq. 2 with finite relative orders $\bar{r}$ and $\bar{\rho}_{j}, j=1, \ldots, \rho$ always has $r=1$ and $\rho_{j}=1, j=1, \ldots, \rho$. Thus, if a discrete-time nonlinear system of the form of Eq. 1 has $r>1$, then the quantity:

$$
\tau_{p} \triangleq(r-1) \Delta t
$$

represents the plant deadtime between the input and the measured output $y$, whereas the additional delay $\Delta t$ is the delay due to sampling.

If a discrete-time nonlinear system of the form of Eq. 1 has $\rho_{j}>1$, then the quantity:

$$
\tau_{d_{j}} \triangleq\left(\rho_{j}-1\right) \Delta t
$$

represents the disturbance deadtime between the measured disturbance $d_{j}$ and the measured output $y$, whereas the additional delay $\Delta t$ is the delay due to sampling.

Therefore, $r$ and $\rho_{j}$ are the smallest number of sampling periods after which the manipulated input move $u(k)$ and the measured disturbance signal $d_{j}(k)$, respectively, affect the measured output signal $y(k)$. As we will see, it is desirable to have $\rho_{j} \geq r\left(\tau_{d_{j}} \geq \tau_{p}\right), j=1, \ldots, \rho$, which means that "early detection" of disturbances (upstream of the process under consideration) is desirable from the point of view of control. This early detection of process disturbances makes the complete elimination of the measurable disturbances possible.

Throughout this article, it is assumed that:
To synthesize a static feedforward/state feedback law that can completely eliminate or at least reject as effectively as possible the effect of measurable disturbances, we must first understand how relative orders are affected by the presence of a feedforward/feedback law of the form of Eq. 3. This issue is addressed in the following theorem.

Theorem 1. Consider a system of the form of Eq. 1 and denote by $r, \rho_{1}, \ldots, \rho_{p}$ the relative orders with respect to $u$, $d_{1}, \ldots, d_{p}$, respectively. Then, under the static feedforward/ state feedback law of Eq. 3, for the closed-loop system (Eq. 7),

(a) the relative order with respect to $v$ is equal to $r$,

(b) the relative order with respect to the disturbance input $d_{j}$ is equal to $\rho_{j}$, if $\rho_{j}<r$ and is greater than or equal to $\rho_{j}$, if $\rho_{j} \geq r$.

The proof is given in the Appendix.

Remark 1. Theorem 1 can be interpreted intuitively as follows. Under the feedforward/state feedback of Eq. 3, the timedelay between the output measurement $y(k)$ and the reference input $v(k)$ is the same as the process deadtime between the output measurement $y(k)$ and the manipulated input $u(k)$. However, the deadtime between the output measurement $y(k)$ and each disturbance measurement signal $d_{j}(k)$ may not be preserved under the feedforward/state feedback law.

\section{Synthesis of Feedforward/State Feedback Laws Feedforward/state feedback law for processes with one measurable disturbance}

To fix the ideas, we consider first systems of the form of Eq. 1 with only one measurable disturbance:

$$
\left\{\begin{aligned}
x(k+1) & =\Phi\left[x(k), u(k), d_{1}(k)\right] \\
y(k) & =h[x(k)]
\end{aligned}\right.
$$

and denote by $r$ the relative order with respect to $u$ and by $\rho_{1}$ the relative order with respect to $d_{1}$.

As we will see in a moment, the nature of the feedforward/ 
feedback control problem will depend upon whether $\rho_{1} \geq r$, or $\rho_{1}<r$. In order to be able to derive the controller synthesis formulas, the following notation is defined.

- When $\rho_{1} \geq r$, it follows from Definitions 1 and 2 that:

$$
h \circ \Phi, h \circ \Phi \circ \Phi, \ldots, h \circ \Phi \circ \ldots \circ \circ \Phi
$$

are functions of $x$ only. Thus, we can define:

$$
\begin{aligned}
h^{1}(x) & \triangleq h\left[\Phi\left(x, u, d_{1}^{\prime}\right)\right] \\
h^{l+1}(x) & \triangleq h^{l}\left[\Phi\left(x, u, d_{1}\right)\right], \quad l=1, \ldots, r-2
\end{aligned}
$$

- When $\rho_{1}<r$, it follows from Definitions 1 and 2 that:

$$
h \circ \Phi, h \circ \Phi \circ \Phi, \ldots, h \circ \frac{\Phi \circ \ldots \circ \Phi}{\left(\rho_{1}-1\right) \text { times }}
$$

are functions of $x$ only, and we can define as before:

$$
\begin{aligned}
h^{\phi}(x) & \triangleq h(x) \\
h^{1}(x) & \triangleq h\left[\Phi\left(x, u, d_{1}\right)\right] \\
h^{l+1}(x) & \triangleq h^{\prime}\left[\Phi\left(x, u, d_{1}\right)\right], \quad l=1, \ldots, \rho_{1}-2
\end{aligned}
$$

On the other hand:

$$
\begin{aligned}
& h \circ \Phi \circ \ldots \circ \Phi, h \circ \Phi \circ \ldots \circ \Phi, \ldots, h \circ \Phi \circ \ldots \circ \Phi \\
& \overline{\rho_{1} \text { times }} \overline{\left(\rho_{1}+1\right) \text { times }}
\end{aligned}
$$

are functions of $x$ and $d$ but independent of $u$. So, we can define:

$$
\begin{aligned}
& h^{\rho_{1}}\left(x, d_{1}\right) \triangleq h^{\rho_{1}-1}\left[\Phi\left(x, u, d_{1}\right)\right] \\
& h^{l+1}\left(x, d_{1}\right) \triangleq h^{\prime}\left[\Phi\left(x, u, d_{1}\right), d_{1}\right], \quad l=\rho_{1}, \ldots, r-2
\end{aligned}
$$

- In either case, $h \circ \underset{r \text { times }}{\Phi \circ \ldots \circ \Phi}$ depends on $u$ as an immediate consequence of Definition 1. This implies that when $\rho_{1} \geq r$, the equation in $u$ :

$$
h^{r-1}\left[\Phi\left(x, u, d_{1}\right)\right]=y^{*}
$$

is locally solvable for $u$ via the implicit function theorem. We will denote by:

$$
u=\Psi_{o}\left(x, d_{1}, y^{*}\right)
$$

the corresponding implicit function. Similarly, when $\rho_{1}<r$, the equation in $u$ :

$$
h^{r-1}\left[\Phi\left(x, u, d_{1}\right), d_{1}\right]=y^{*}
$$

is locally solvable for $u$ via the implicit function theorem. We will denote by:

$$
u=\Psi_{o}\left(x, d_{1}, y^{*}\right)
$$

the corresponding implicit function (note that we use the same symbol $\Psi_{o}$ for the solutions of Eqs. 12 and 14 for notational convenience).

We are now in a position to pose and solve the following problem:

For a system of the form of Eq. 8, synthesize a feedforward/ state feedback law of the form:

$$
u(k)=\Psi\left[x(k), d_{1}(k), v(k)\right]
$$

so that in closed-loop, $y$ is unaffected by $d$, and $y$ depends linearly on $v$.

Theorem 2. The problem $(P I)$ is solvable, if and only if $\rho_{1} \geq r$. For systems with $\rho_{1} \geq r$, the feedforward/state feedback law:

$$
\begin{aligned}
u(k)= & \Psi_{0}\left(x(k), d_{1}(k),\left(1+\sum_{l=1}^{r} \beta_{l}\right) v(k)\right. \\
& \left.-\sum_{l=1}^{r} \beta_{l} h^{r-l}(x(k))\right)
\end{aligned}
$$

where $\Psi_{0}(., \ldots$, is defined by Eqs. 12 and 13, induces the linear input/output dynamics:

$$
y(k+r)+\sum_{l=1}^{r} \beta_{l} y(k+r-l)=\left(1+\sum_{l=1}^{r} \beta_{l}\right) v(k)
$$

to the closed-loop v-y system. The proof is given in the Appendix.

Remark 2. The term $\left(1+\Sigma_{l=1}^{r} \beta_{l}\right)$ is used as the coefficient of $v(k)$ in the control law of Eq. 16 and the closed-loop response of Eq. 17 to enforce unit static gain between $v$ and $y$.

Remark 3. For a process of the form of Eq. 8 with $\rho_{1} \geq r$, the closed-loop response under a feedforward/state feedback law of Eq. 16 is shaped only by the choice of the tuning parameters $\beta_{l}$.

For instance:

(i) If we choose the tuning parameters $\beta_{l}$ according to $\beta_{1}=\ldots=\beta_{r}=0$, the feedforward/state feedback law of Eq. 16 will simplify to:

$$
u(k)=\Psi_{o}\left(x(k), d_{1}(k), v(k)\right),
$$

which induces the deadbeat (integral-square error (ISE)-optimal) response:

$$
y(k+r)=v(k)
$$

to the closed-loop $v-y$ system. In practice, deadbeat response will not be requested despite its ISE-optimality. This is because of the poor robustness of deadbeat control. A convenient type 
of response in practice is the "first-order-plus-deadtime," which is obtained by setting the parameters $\beta_{l}$ as given below.

(ii) If we choose the tuning parameters $\beta_{l}$ according to $\beta_{2}=\ldots=\beta_{r}=0$ and $\beta_{1}=-\alpha$ with $0<\alpha<1$ tunable, the feedforward/state feedback law of Eq. 16 will simplify into:

$$
u(k)=\Psi_{o}\left\{x(k), d_{1}(k),(1-\alpha) v(k)+\alpha h^{r-1}[x(k)]\right\}
$$

which induces the linear input/output dynamics (first-orderplus-deadtime response):

$$
y(k+r)-\alpha y(k+r-1)=(1-\alpha) v(k)
$$

to the closed-loop $v$ - $y$ system.

Since problem $(\mathrm{P} 1)$ is only solvable when $\rho_{1} \geq r$, one must pose and solve a weaker problem for the situation $\rho_{1}<r$. Since complete elimination of the disturbance is not possible, we must look for some optimal type response that can be induced by feedforward/state feedback. Our intuition from linear systems tells us that optimal selection of the control law will strongly depend on the nature of changes in $v$ and/or $d_{1}$, that is, whether they are steps, ramps, exponentials, and so on. In what follows, we will restrict our attention to steps and formulate and solve the following problem:

For a system of the form of Eq. 8 with $\rho_{1}<r$, synthesize a feedforward/state feedback law of the form:

$$
u(k)=\Psi\left[x(k), d_{1}(k), v(k)\right]
$$

so that in closed-loop step changes in $d$ are optimally rejected, and step changes in $v$ are optimally followed in the sense of the ISE criterion.

Theorem 3. Consider a system of the form of Eq. 8 with $\rho_{1}<r$ and the feedforward/state feedback law:

$$
\begin{aligned}
u(k) & =\Psi_{o}\left(x(k), d_{1}(k),\left(1+\sum_{l=1}^{r} \beta_{l}\right) v(k)\right. \\
& \left.-\sum_{l=1}^{r-\rho_{1}} \beta_{l} h^{r-l}\left(x(k), d_{1}(k)\right)-\sum_{l=r \sim \rho_{1}+1}^{r} \beta_{l} h^{r-l}(x(k))\right)
\end{aligned}
$$

Then, for $\beta_{1}=\ldots=\beta_{r}=0$, the resulting closed-loop dynamics induces ISE-optimal response to step changes in either $v$ or $d_{1}$. The proof is given in the Appendix.

Note that for systems with $\rho_{1}<r$, the complete elimination of the disturbance is not possible and the control law of Eq. 16 does not, in general, induce a linear response in either $v$ or $d_{1}$.

Remark 4. Although the parameter settings $\beta_{1}=\ldots=\beta_{r}=0$ lead to ISE-optimal response to step changes in $v$ and $d_{1}$, for robustness reasons, these settings will not be used in practice. One would probably want to use $\beta_{2}=\ldots=\beta_{r}=0$ and $\beta_{1}=-\alpha$ with $0<\alpha<1$ tunable. These settings correspond to a feedforward/state feedback law of the form:

$$
u(k)=\Psi_{o}\left(x(k), d_{1}(k),(1-\alpha) v(k)+\alpha h^{r-1}\left(x(k), d_{1}(k)\right)\right)
$$

Feedforward/state feedback law for processes with measurable disturbances

We are now in a position to generalize the one-disturbance controller synthesis results to the general multidisturbance case. In this direction, the set of disturbances is partitioned into three classes:

$$
\begin{aligned}
& d_{j} \in \mathbb{Q} \Leftrightarrow \rho_{j}>r \\
& d_{j} \in \mathbb{B} \Leftrightarrow \rho_{j}=r \\
& d_{j} \in \mathcal{C} \Leftrightarrow \rho_{j}<r
\end{aligned}
$$

and denote by $d_{Q}$ the vector of the disturbances in class $Q, d_{\mathscr{B}}$ the vector of the disturbances in class $B$ and $d_{\mathrm{e}}$ the vector of the disturbances in class $\mathfrak{C}$.

The partitioning of measurable disturbances into above classes can be intuitively supported by the following considerations:

- If a disturbance $d_{j}$ is in the class $Q$, the disturbance deadtime $\tau_{d_{j}}$ will be greater than the plant deadtime $\tau_{p}$.

- If a disturbance $d_{j}$ is in the class $\mathcal{B}$, the disturbance deadtime $\tau_{d}$, will be equal to the plant deadtime $\tau_{p}$.

- If a disturbance $d_{j}$ is in the class $\mathcal{C}$, the disturbance deadtime $\tau_{d}$, will be smaller than the plant deadtime $\tau_{p}$.

If transportation-lag contributes to the time-delay of a disturbance in class $\mathcal{C}$ or $\mathbb{B}$, one may be able to convert this disturbance into a $\mathbb{B}$ - or $\mathbb{Q}$-type disturbance by moving the disturbance sensor farther upstream of the process. On the other hand, introduction of measurement time-delay to a disturbance measurement can change a $Q$-type or $B$-type disturbance to a $ß$-type or $\mathcal{C}$-type disturbance; a measurement time-delay in a disturbance measurement may prevent the complete elimination of the disturbance. Therefore, measurement time-delays in the disturbance measurements have a negative effect on achievable control quality, which is in complete agreement with our understanding from linear control theory.

The notation $h^{\mathrm{t}}, h^{2}, \ldots, h^{r-1}$ was defined for systems with one measurable disturbance. Here, we need to extend this notation for systems with measurable disturbances. In doing so, we explicitly indicate that these notations depend on $x$ and the disturbances of class $\mathcal{C}$ only, as:

$$
\begin{aligned}
h^{\prime}\left(x, d_{\mathrm{e}}\right) & \triangleq h\left[\Phi\left(x, u, d_{1}, \ldots, d_{p}\right)\right] \\
h^{l+1}\left(x, d_{\mathrm{e}}\right) & \triangleq h^{l}\left[\Phi\left(x, u, d_{1}, \ldots, d_{p}\right), d_{\mathrm{C}}\right], \quad l=1, \ldots, r-2
\end{aligned}
$$

Then, the equation in $u$ :

$$
h^{r-1}\left(\Phi\left(x, u, d_{1}, \ldots, d_{p}\right), d_{\mathrm{C}}\right)=y^{*}
$$

will be locally solvable for $u$ via the implicit function theorem. We will denote by:

$$
u=\Psi_{o}\left(x, d_{\infty}, d_{e}, y^{*}\right)
$$

the corresponding implicit function. Here we explicitly indicate that $\Psi_{o}$ will depend on disturbances of classes $B$ and $\mathcal{C}$ but not on disturbances of class $Q$. 
Theorem 4. Consider a system of the form of Eq. 1 whose set of disturbance inputs has been partitioned into classes $Q$, $B$ and $\mathcal{C}$ according to Eq. 19:

$$
\left\{\begin{aligned}
x(k+1) & =\Phi\left[x(k), u(k), d_{\mathbb{Q}}(k), d_{\mathbb{B}}(k), d_{\mathcal{C}}(k)\right] \\
y(k) & =h[x(k)]
\end{aligned}\right.
$$

Then, the feedforward/state feedback law:

$$
\begin{aligned}
u(k)=\Psi_{o}\left(x(k), d_{B \mathrm{~B}}(k), d_{\mathrm{C}}(k),\left(1+\sum_{l=1}^{r} \beta_{l}\right) v(k)\right. \\
\left.-\sum_{l=1}^{r} \beta_{l} h^{r-l}\left[x(k), d_{\mathrm{e}}(k)\right]\right)
\end{aligned}
$$

where $\Psi_{0}(., \ldots,$.$) is defined by Eqs. 21$ and 22:

(a) completely rejects the effect of the disturbances of classes $\mathbb{Q}$ and $B$ on the output $y$; and

(b) provides ISE-optimal rejection of the effect of step changes in disturbances in class $\mathcal{C}$ on the output $y$ for $\beta_{1}=\ldots=\beta_{r}=0$. The proof is given in the Appendix.

The relative magnitude of the plant deadtime $\left(\tau_{p}\right)$ with respect to the disturbance deadtimes $\left(\tau_{d}, j=1, \ldots, p\right)$ characterizes the dependence of the feedforward/state feedback law of Eq. 24 on the disturbance measurements; the feedforward/ state feedback of Eq. 24 is a function of the past measurements of the Q-type disturbances, and the present measurements of the $B$ - and $\mathcal{C}$-type disturbances.

Remark 5. If there is no disturbance $d_{j}$ with $\rho_{j}<r$ (class $\mathfrak{C}=\phi$ ), the feedforward/state feedback of Theorem 4 will completely eliminate the effect of measurable disturbances and will induce the linear response:

$$
y(k+r)+\sum_{l=1}^{r} \beta_{l} y(k+r-l)=\left(1+\sum_{l=1}^{r} \beta_{l}\right) v(k)
$$

to the closed-loop $v-y$ system.

The above results suggest that it is desirable to have $\rho_{j} \geq r$, $j=1, \ldots \rho$, that is, to have disturbance measurement signals to enter the process with longer or equal time-delays compared to the plant deadtime $\left(\tau_{p}\right)$. In this case, the measurable disturbances can be rejected completely.

Remark 6. In the case that there is no disturbance measurement, the feedforward/feedback law of Eq. 24 will become a pure feedback law and will be exactly the state feedback given in (Soroush and Kravaris, 1992a).

\section{Minimum-Phase Behavior in Discrete-Time Sys- tems with Disturbances and Conditions for Asymp- totic Stability of the Closed-Loop System}

For disturbance-free discrete-time nonlinear systems, Monaco and Normand-Cyrot (1988) introduced a notion of zero dynamics that allows a characterization of minimum-phase and nonminimum-phase behavior. The notion of zero dynamics is based on a local normal form defined on the zero-outputconstrained manifold. It is then shown that the delay-free part of a nonlinear system is minimum phase, if and only if the closed-loop dynamics induced by a deadbeat controller is stable.
In what follows, a notion of minimum-phase-ness for nonlinear systems with disturbances will be introduced. This notion will be in the same spirit of the disturbance-free case but will not involve the definition of a normal form. From the results of the previous section, it follows that the feedforward/state feedback law:

$$
u(k)=\Psi_{o}\left[x(k), d_{\mathbb{B}}(k), d_{\mathrm{C}}(k), v(k)\right]
$$

induces a deadbeat response in $v$ whenever the disturbances remain unchanged. Thus, the dynamics of the resulting closedloop system can be used for a characterization of minimumphase behavior.

Definition 3. A system of the form of Eq. 23 is said to have stable finite zero dynamics, if the dynamic system:

$$
\begin{array}{r}
x(k+1)=\Phi\left\{x(k), \Psi_{o}\left[x(k), d_{\mathbb{B}}(k), d_{\mathcal{C}}(k), v(k)\right],\right. \\
\left.d_{\mathbb{Q}}(k), d_{\mathbb{B}}(k), d_{\mathcal{C}}(k)\right\}
\end{array}
$$

is locally asymptotically stable. Otherwise, it is said to have unstable finite zero dynamics.

The local asymptotic stability of the system of Eq. 26 can be checked by calculating the eigenvalues of the Jacobian matrix of the system evaluated at a nominal equilibrium point. Using the definition of $\Psi_{o}$ (Eqs. 21 and 22), we obtain the Jacobian matrix of the above system (Eq. 26):

$$
\begin{aligned}
& J_{o}(x, u, d)=\left(\frac{\partial \Phi(x, u, d)}{\partial x}\right)-\left(\frac{\partial \Phi(x, u, d)}{\partial u}\right) \\
& \left.\left.\times\left(\frac{\partial}{\partial x} h^{r-1}(\Phi(x, u, d), d)\right)\right)\left(\frac{\partial}{\partial u} h^{r-1}(\Phi(x, u, d), d)\right)\right)^{-1}
\end{aligned}
$$

If, at the given equilibrium point, all the eigenvalues of $\oiint_{o}(x$, $u, d)$ are in the interior of the unit circle, the dynamics of Eq. 26 will be guaranteed to be locally asymptotically stable around that point.

Definition 4. A system of the form of Eq. 23 is said to have hyperbolically stable finite zero dynamics, if all the eigenvalues of the Jacobian matrix given by Eq. 27, evaluated at the nominal equilibrium point, lie in the interior of the unit circle.

With the above definitions, we are now in a position to study the local asymptotic stability of the closed-loop dynamics induced by the feedforward/feedback law of Eq. 24 .

Theorem 5. Consider a system of the form of Eq. 23, which is subject to the feedforward/state feedback law of Eq. 24. Then, the dynamics of the closed-loop system, that is:

$$
\begin{gathered}
x(k+1)=\Phi\left(x(k), \Psi_{o}\left(x(k), d_{\mathbb{B}}(k), d_{\mathrm{e}}(k),\right.\right. \\
\left.\left(1+\sum_{i=1}^{r} \beta_{l}\right) v(k)-\sum_{l=1}^{r} \beta_{i} h^{r-l}\left[x(k), d_{\mathrm{e}}(k)\right]\right), \\
\left.d_{\mathbb{Q}}(k), d_{\mathbb{B}}(k), d_{\mathrm{e}}(k)\right)
\end{gathered}
$$$$
y(k)=h(x(k))
$$ 
is locally asymptotically stable, if the following conditions are met:

(i) The system of Eq. 23 has hyperbolically stable finite zero dynamics; and

(ii) the roots of the characteristic equation:

$$
z^{r}+\beta_{1} z^{r-1}+\ldots+\beta_{r-1} z+\beta_{r}=0
$$

lie inside the unit circle.

The proof is given in the Appendix.

\section{Synthesis of Feedforward/Dynamic Feedback Controllers}

The theoretical results of the previous section provided synthesis formula for design of the static feedforward/state feedback laws, under which the class of measurable disturbances can be rejected as effectively as possible, and a desirable linear input/output closed-loop response may be induced to the process under consideration. The resulting feedforward/state feedback structure is depicted in Figure 1.

To reject asymptotically the unmeasurable disturbances and to ensure the offsetless tracking of output set-point changes, we can use an error feedback controller with integral action around the $v-y$ loop. The resulting control structure (the feedforward/feedback law plus the error feedback controller) is depicted in Figure 2 and is the feedforward GLC structure. In the case the $v-y$ system is linear, if we use a linear error feedback controller with integral action around the $v-y$ loop, the overall closed-loop system will also be linear. This linear error feedback controller will be referred to as the external controller (Kravaris and Chung, 1987). The linearity of the resulting closed-loop $y_{s p}-y$ system facilitates greatly the characterization and the study of the theoretical properties of the closed-loop system.

\section{External controller}

As shown earlier, if there is no disturbance with $\rho_{j}<r(\mathcal{E}=\phi)$, the state feedback of Eq. 24 induces the linear input/output behavior of Eq. 25 to the closed-loop system. In this case, if we use a linear error feedback controller with the transfer function:

$G_{e}(z)=\left(\begin{array}{l}1+\sum_{l=1}^{r} \gamma_{i} \\ 1+\sum_{i=1}^{r} \beta_{l}\end{array}\right)\left(\frac{z^{r}+\beta_{1} z^{r-1}+\ldots+\beta_{r-1} z+\beta_{r}}{z^{r}-1+\gamma_{1}\left(z^{r-1}-1\right)+\ldots+\gamma_{r-1}(z-1)}\right)$

where $\gamma_{l}, l=1, \ldots, r$ are adjustable scalar parameters, around the $v-y$ system, the resulting overall closed-loop system will be:

$$
y(k+r)+\sum_{l=1}^{r} \gamma_{l} y(k+r-l)=\left(1+\sum_{l=1}^{r} \gamma_{l}\right) y_{s p}(k)
$$

Note that $G_{e}(z)$ has a pole at $z=1$; the external controller possesses integral action which guarantees the offsetless tracking of set-point changes.
Minimal-order state-space realizations of the transfer function of Eq. 29 can be found in standard linear systems literature (Isermann, 1989). For example, the state-space realization:

$$
\begin{aligned}
\xi(k+1) & =A_{e} \xi(k)+b_{e} e(k) \\
\left(1+\sum_{l=1}^{r} \beta_{l}\right) v(k) & =c_{e} \xi(k)+f_{e} e(k)
\end{aligned}
$$

where $\xi(k)=\left[\xi_{1}(k) \ldots \xi_{r}(k)\right]^{T} \in \mathbb{R}^{r}$ and:

$$
\begin{aligned}
& A_{e}=\left[\begin{array}{ccccc}
-\gamma_{1} & -\gamma_{2} & \ldots & -\gamma_{r-1} & \left(1+\gamma_{1}+\ldots+\gamma_{r-1}\right) \\
1 & 0 & \ldots & 0 & 0 \\
0 & 1 & \ldots & 0 & 0 \\
\vdots & \vdots & \ddots & \vdots & \vdots \\
0 & 0 & \ldots & 1 & 0
\end{array}\right], \\
& b_{e}=\left[\begin{array}{c}
1+\gamma_{1}+\ldots+\gamma_{r} \\
0 \\
0 \\
\vdots \\
0
\end{array}\right] \\
& c_{e}=\left[\left(\beta_{1}-\gamma_{1}\right)\left(\beta_{2}-\gamma_{2}\right) \ldots\right. \\
&\left.\left(\beta_{r-1}-\gamma_{r-1}\right)\left(1+\gamma_{1}+\ldots+\gamma_{r-1}+\beta_{r}\right)\right]
\end{aligned}
$$

and $f_{e}=1+\gamma_{1}+\ldots+\gamma_{r}$, is a minimal-order state-space realization of the transfer function $G_{e}(z)$ defined by Eq. 29 .

Remark 7. For a dead-time-free process $(r=1)$, the transfer function $G_{e}(z)$ defined by Eq. 29 simplifies to:

$$
G_{e}(z)=\left(\frac{1+\gamma_{1}}{1+\beta_{1}}\right)\left(\frac{z+\beta_{1}}{z-1}\right)
$$

which is exactly a digital proportional integral (PI) controller. The use of PI controllers to control a linear $v-y$ system may be sufficient in many applications (even for $r>1$ ), although in general, one must sacrifice performance for the sake of simplicity of the external controller. In the case $r=1$, the realization of Eq. 31 simplifies into:

$$
\begin{aligned}
\xi_{1}(k+1) & =\xi_{1}(k)+\left(1+\gamma_{1}\right) e(k) \\
v(k) & =\xi_{1}(k)+\frac{1+\gamma_{1}}{1+\beta_{1}} e(k)
\end{aligned}
$$

which is a minimal-order realization of the above PI controller transfer function.

\section{Synthesis of feedforward/dynamic mixed-error and state-feedback controllers}

In the case that all the process state variables are measured on-line, the feedforward/state feedback (Eq. 24) in conjunc- 
tion with the external controller (Eq. 31) is implemented, leading to the control structure shown in Figure 2. Precise formulation of this idea is given in the following theorem.

Theorem 6. Consider a nonlinear process of the form of Eq. 23. Then, the dynamic system:

$$
\left\{\begin{array}{r}
\xi(k+1)=A_{e} \xi(k)+b_{e} e(k) \\
u(k)=\Psi_{o}\left(x(k), d_{\mathbb{B}}(k), d_{e}(k), c_{e} \xi(k)+f_{e} e(k)\right. \\
\left.-\sum_{l=1}^{r} \beta_{l} h^{r-1}\left[x(k), d_{e}(k)\right]\right)
\end{array}\right.
$$

where $\Psi_{o}(., \ldots, \ldots)$ is defined by Eqs. 21 and 22, and $A_{e}, b_{e}$, $c_{e}$ and $f_{e}$ are the system matrices of the realization of $E q .31$, represents an rth order state-space realization of a feedforward/dynamic mixed error-and state-feedback controller that induces the closed-loop input/output behavior described by $E q .30$, if $\rho_{j} \geq r, j=1, \ldots, p$. The proof is given in the Appendix.

The block diagram of the feedforward/dynamic mixed errorand state-feedback controller (Eq. 32) is depicted in Figure 2. This control structure is suitable when all the state variables $x$ can be measured on-line.

\section{Synthesis of feedforward/dynamic error feedback controllers for open-loop stable processes}

In the case the on-line measurements of states are not available and the process is open-loop stable, an open-loop observer can be employed to reconstruct the states. This approach involves on-line simulation of the process model:

$$
w(k+1)=\Phi\left[w(k), u(k), d_{1}(k), \ldots, d_{p}(k)\right]
$$

where $w$ represents the vector of the estimated states, driven by the manipulated input and measurements of the measurable disturbances.

The state estimates $w$ can be used in the feedforward/state feedback of Eq. 24, that is:

$$
\begin{aligned}
u(k)=\Psi_{o}\left(w(k), d_{\mathrm{B}}(k),\right. & d_{\mathrm{e}}(k),\left(1+\sum_{l=1}^{r} \beta_{l}\right) v(k) \\
& \left.-\sum_{l=1}^{r} \beta_{l} h^{r-l}\left[w(k), d_{\mathrm{e}}(k)\right]\right)
\end{aligned}
$$

where $v$ is the output of the external controller of Eq. 31 . Combining Eqs. 31, 33 and 34, we obtain a state-space realization of a controller, which induces the requested closedloop dynamics (Eq. 30) if $\rho_{j} \geq r, j=1, \ldots, p$. This idea is formulated precisely in the theorem that follows.

Theorem 7. Consider a nonlinear process of the form of Eq. 23. Then, the dynamic system:

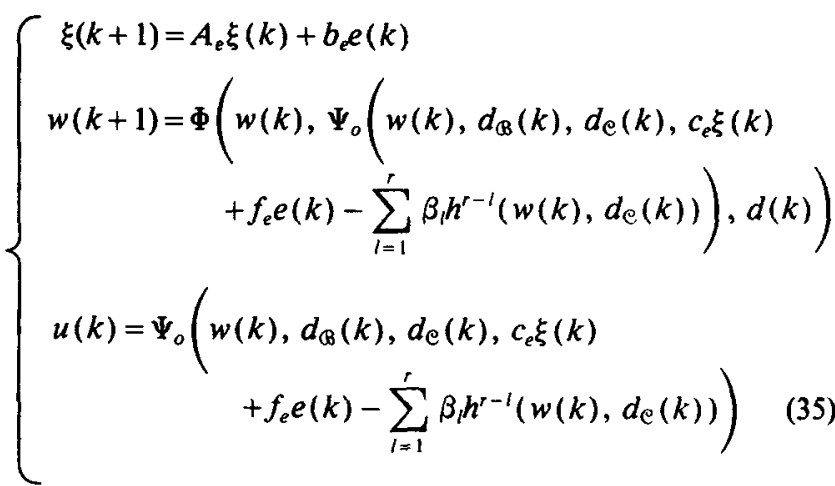

where $\Psi_{o}(., \ldots, \ldots)$ is defined by Eqs. 21 and 22 , and $A_{e}, b_{e}$, $c_{e}$ and $f_{e}$ are the system matrices of the realization of $E q .31$, represents an $(n+r)$ th order state-space realization of a feedforward/dynamic error-feedback controller that induces the closed-loop input/output behavior of Eq. 30, if $\rho_{j} \geq r, j=1$, $\ldots, p$. The proof is given in the Appendix.

Corollary 1 (Reduced-order realization). Under the assumptions of Theorem 7, the dynamic system:

$$
\left\{\begin{array}{c}
w(k+1)=\Phi\left(w(k), \Psi_{o}\left(w(k), d_{\mathbb{B}}(k), d_{\mathrm{e}}(k),\right.\right. \\
\left(1+\sum_{l=1}^{r} \gamma_{l}\right)(e(k)+h(w(k))) \\
\left.\left.-\sum_{l=1}^{r} \gamma_{l} h^{r-1}\left(w(k), d_{\mathrm{e}}(k)\right)\right), d(k)\right) \\
u(k)=\Psi_{o}\left(w(k), d_{\mathbb{B}}(k), d_{\mathrm{e}}(k),\right. \\
\left(1+\sum_{l=1}^{r} \gamma_{l}\right)(e(k)+h(w(k))) \\
\left.-\sum_{l=1}^{r} \gamma_{l} h^{r-1}\left(w(k), d_{\mathrm{e}}(k)\right)\right)
\end{array}\right.
$$

represents an nth order realization of a feedforward/dynamic error-feedback controller that induces the closed-loop input/ output behavior of Eq. 30, if $\rho_{j} \geq r, j=1, \ldots, p$. The proof is given in the Appendix.

The controller realizations (Eqs. 35 and 36) represent a feedforward/dynamic error-feedback controller with integral action. The overall control structure as well as the various parts of the controller of Eq. 35 are shown in Figure 3a. The control structure of the reduced-order controller (Eq. 36) is depicted in Figure 3b.

Remark 8. From a practical point of view, it is desirable to have a minimum number of controller parameters and at the same time enough flexibility to "shape" the closed-loop response. A popular approach is to place $(r-1)$ poles of the closed-loop system at the origin and leaving the remaining pole adjustable. This pole placement corresponds to choosing:

$$
\gamma_{2}=\ldots=\gamma_{r}=0, \quad \gamma_{1}=-\alpha, \quad 0<\alpha<1
$$

For example, the controller of Eq. 36 with the settings of Eq. 37 simplifies to: 


$$
\left\{\begin{array}{r}
w(k+1)=\Phi\left\{w(k), \Psi_{o}\left\{w(k), d_{\mathbb{B}}(k), d_{\mathcal{C}}(k),\right.\right. \\
\left.\left.(1-\alpha)[e(k)+h(w(k))]+\alpha h^{r-1}\left[w(k), d_{\mathbb{C}}(k)\right]\right\}, d(k)\right\} \\
u(k)=\Psi_{o}\left\{w(k), d_{\mathbb{B}}(k), d_{\mathbb{C}}(k),(1-\alpha)[e(k)+h(w(k))]\right. \\
\left.+\alpha h^{r-1}\left[w(k), d_{\mathbb{C}}(k)\right]\right\}
\end{array}\right.
$$

This controller, when is applied to processes with $\rho_{j} \geq r, j=1$, $\ldots, p$, induces the first-order-plus-deadtime response:

$$
y(k+r)-\alpha y(k+r-1)=(1-\alpha) y_{s p}(k)
$$

or in $z$-domain:

$$
\frac{y(z)}{y_{s p}(z)}=z^{-r} \frac{1-\alpha}{1-\alpha z^{-1}}
$$

to the overall closed-loop system.

Later it will be shown that when the controller synthesis formula of Eq. 38 is applied to linear systems, the resulting linear controller will be exactly a minimal-order state-space realization of the feedforward IMC (Garcia and Morari, 1985).

Remark 9. In general, a controller with reduced-order openloop state observer provides a better performance (especially in the presence of modeling errors and unmeasurable disturbances) than a controller with full-order open-loop state observer. On-line process information should be used to infer as many states as possible, and therefore, impiementing an observer with the lowest possible order. This reduced-order openloop state observer should be implemented in conjunction with the controller of Theorem 6 (Eq. 32). The use of reduced-order open-loop observers has been suggested for the processes that are open-loop unstable and/or for processes whose states cannot all be measured on-line (Soroush and Kravaris, 1992a).

\section{Closed-loop stability}

Input/Output Stability. The input/output stability of the overall closed-loop system (under the controllers of Theorems 6 and 7 and Corollary 1) will be guaranteed, if the condition:

(i) $\lambda T / \lambda$ the parameters $\gamma_{l}, l=1, \ldots, r$ are chosen so that the roots of the characteristic equation:

$$
z^{r}+\gamma_{1} z^{r-1}+\ldots+\gamma_{r-1} z+\gamma_{r}=0
$$

lie inside the unit circle.

Internal Stability. In addition to the input/output stability, one must assure that the overall closed-loop system is internally stable, that is, the state variables always remain bounded. Using Lyapunov's first method, one can show that if the overall closed-loop system is input/output stable and in addition the following conditions are satisfied: ics.

(ii) The process has hyperbolically stable finite zero dynam-

(iii) The parameters $\beta_{i}, l=1, \ldots, r$ are chosen so that the roots of the characteristic equation of Eq. 28 lie inside the unit circle.

Then the local internal stability of the closed-loop system under the controller of Theorem 6 will be guaranteed. Con- ditions for internal stability of the closed-loop system under the controllers of Theorem 7 and Corollary 1 are as follows:

- The controller of Theorem 7: The conditions (i), (ii), (iii) and local asymptotic stability of the open-loop process.

- The controller of Corollary 1: The conditions (i), (ii) and local asymptotic stability of the open-loop process.

\section{Feedforward/Dynamic Error.Feedback Control of Linear Systems}

Consider SISO time-invariant discrete-time linear systems described by a state space model of the form:

$$
\left\{\begin{aligned}
x(k+1) & =A x(k)+b u(k)+\sum_{j=1}^{p} \zeta_{j} d_{j}(k) \\
y(k) & =c x(k)
\end{aligned}\right.
$$

where $A, b$ and $c$ are $n \times n, n \times 1$ and $1 \times n$ constant matrices, respectively. Here $\zeta_{1}, \ldots, \zeta_{p}$ are $n \times 1$ constant matrices. The above model is a special case of Eq. 1 for:

$$
\begin{gathered}
\Phi\left[x(k), u(k), d_{1}(k), \ldots, d_{p}(k)\right]=A x(k)+b u(k) \\
+\sum_{j=1}^{p} \zeta_{j} \mathrm{~d}_{j}(\mathrm{k}) \\
h[x(k)]=c x(k)
\end{gathered}
$$

Applying Definitions 1 and 2 to the system of Eq. 40, we immediately see that the relative order $r$ is the smallest integer for which $c A^{r-1} b \neq 0$, and the relative order $\rho_{j}$ is the smallest integer for which $c A^{\rho_{j-1}} \zeta_{j} \neq 0$. Furthermore, the following relations hold:

$$
\begin{gathered}
h^{i}\left(x, d_{\mathcal{E}}\right)=c A^{i} x+\sum_{j=1}^{p} \sum_{l=0}^{i-\rho_{j}} c A^{i-1-1} \zeta_{j} d_{j}, \quad i=0, \ldots, r-1 \\
h^{r-1}\left(\Phi\left(x, u, d_{1}, \ldots, d_{p}\right), d_{\mathcal{C}}\right)=c A^{r} x \\
+c A^{r-1} b u+\sum_{j=1}^{p} \sum_{l=0}^{r-\rho_{j}} c A^{r-1-1} \zeta_{j} d_{j}
\end{gathered}
$$

and the function $\Psi_{o}$, which was defined implicitly as the solution of Eq. 21, has the simple closed-form expression:

$$
\Psi_{o}\left(x, d_{\mathbb{B}}, d_{\mathrm{e}}, y^{*}\right)=\frac{1}{c A^{r-1} b}\left(y^{*}-c A^{r} x-\sum_{j=1}^{p} \sum_{i=0}^{r-\rho_{j}} c A^{r-l-1} \zeta_{j} d_{j}\right)
$$

The input/output behavior of the system of Eq. 40 can be represented by the $z$-domain transfer function:

$$
y(z)=c(z I-A)^{-1} b u(z)+c(z I-A)^{-1} \sum_{j=1}^{p} \zeta_{j} d_{j}(z) .
$$

Using the algebraic identities:

$$
\begin{gathered}
z^{r} c(z I-A)^{-1} b=c A^{r}(z I-A)^{-1} b+c A^{r-1} b, \\
z^{\rho_{j}} c(z I-A)^{-1} \zeta_{j}=c A^{\rho_{j}}(z I-A)^{-1} \zeta_{j}+c A^{\rho_{j}-1} \zeta_{j},
\end{gathered}
$$


Eq. 42 can be rewritten in the form:

$$
y(z)=z^{-r} H(z) u(z)+\sum_{j=1}^{p} z^{-\rho_{j}} H_{d_{j}}(z) d_{j}(z)
$$

where

$$
\begin{gathered}
H(z)=c A^{\prime}(z I-A)^{-1} b+c A^{r-1} b, \\
H_{d_{j}}(z)=c A^{\rho_{j}}(z I-A)^{-1} \zeta_{j}+c A^{\rho_{j}-1} \zeta_{j} .
\end{gathered}
$$

Equation 45 provides a factorization of the transfer functions $c(z I-A)^{-1} b$ and $c(z I-A)^{-1} \zeta_{j}, j=1, \ldots, p$ input pure delay and delay-free parts in terms of the system matrices $A, b, \zeta_{1}$, $\ldots, \zeta_{p}$ and $c$.

The simplicity of the expressions for $\Phi, h^{\prime}$ and $\Psi_{o}$ in linear systems significantly simplifies the expressions for the derived controllers (Eqs. 32, 35 and 36). In particular, the feedforward/ error-feedback controller of Corollary 1 (Eq. 36) under the choice $\gamma_{1}=-\alpha, \gamma_{2}=\ldots=\gamma_{r}=0$ (which corresponds to requesting first-order-plus-deadtime response), becomes (see also Eq. 38):

$$
\frac{y(z)}{y_{s p}(z)}=z^{-\prime} \frac{1-\alpha}{1-\alpha z^{-1}}
$$

The controller of Eqs. 48 and 49 induces the linear response of Eq. 50 to the closed-loop system (completely eliminates the effect of measurable disturbances), if $\rho_{j} \geq r, j=1, \ldots, m$. If there are disturbances with $\rho_{j}<r$, the best we can do is to set $\alpha=0$, and therefore, optimally reject the $\mathcal{C}$-type disturbances. The following remark will further clarify this point.

Remark 10. Consider linear systems with only one measurable disturbance $(p=1)$ of the type $\mathcal{C}$. In this case, the controller of Eq. 49 with the choice $\alpha=0$ simplifies to:

$$
u(z)=\frac{1}{1-z^{-r}} \frac{1}{H(z)}\left(e(z)-\left(1-z^{-\rho_{1}}\right) H_{d_{1}}(z) d_{1}(z)\right)
$$

which induces the closed-loop response:

$$
y(z)=z^{-r} y_{s p}(z)+z^{-\rho_{1}}\left(1-z^{-\left(r-\rho_{1}\right)}\right) H_{d_{1}}(z) d_{1}(z)
$$

This closed-loop response shows that the controller of Eq. 51 provides ISE-optimal tracking of set-point changes (deadbeat

$$
\left\{\begin{array}{r}
w(k+1)=\left(A-b \frac{c A^{r}-\alpha c A^{r-1}-(1-\alpha) c}{c A^{r-1} b}\right) w(k)+b\left(\frac{1-\alpha}{c A^{r-1} b}\right) e(k) \\
-\frac{\sum_{j=1}^{p}\left[\sum_{l=0}^{r-\rho_{j}} b c A^{r-1-1}-\alpha \sum_{l=0}^{r-\rho_{j}-1} b c A^{r-l-2}-c A^{r-1} b\right] \zeta_{j} d_{j}(k)}{c A^{r-1} b} \\
u(k)=-\left(\frac{c A^{r}-\alpha c A^{r-1}-(1-\alpha) c}{c A^{r-1} b}\right) w(k)+\left(\frac{1-\alpha}{c A^{r-1} b}\right) e(k) \\
-\frac{\sum_{j=1}^{p}\left(\sum_{l=0}^{r-\rho_{j}} c A^{r-l-1}-\alpha \sum_{l=0}^{r-\rho_{j}-1} c A^{r-l-2}\right) \zeta_{j} d_{j}(k)}{c A^{r-1} b}
\end{array}\right.
$$

Equation 48 is a minimal-order state-space realization of the $z$-domain transfer function:

$$
\begin{gathered}
u(z)=\frac{1-\alpha}{1-\alpha z^{-1}-(1-\alpha) z^{-r}} \frac{1}{H(z)}\left[e(z)-\sum_{j=1, d_{j} \in \mathrm{e}}^{p} H_{d_{j}}(z) d_{j}(z)\right. \\
-\frac{1-\alpha z^{-1}}{1-\alpha} \sum_{j=1, d_{j} \in Q, B}^{p} z^{-\left(\rho_{j}-r\right)} H_{d_{j}}(z) d_{j}(z) \\
\left.+\sum_{j=1}^{p} z^{-\rho_{j}} H_{d_{j}}(z) d_{j}(z)\right]
\end{gathered}
$$

where $H(z)$ and $H_{d_{f}}(z)$ are given by Eqs. 46 and 47 . The transfer function of Eq. 49 is exactly the controller derived from the feedforward IMC (Garcia and Morari, 1985; Morari and Zafiriou, 1989), for a requested closed-loop response of the form: control) and ISE-optimal rejection of the disturbance $d_{1}$ (Morari and Zafiriou, 1989).

\section{Model Algorithmic Control (MAC)}

MAC (Richalet et al., 1978; Mehra and Rouhani, 1980) is a model predictive controller whose control horizon is one, prediction horizon is the relative order $r$ (one sampling period beyond the plant deadtime), and move $u(k)$ is obtained from the minimization of square of the deviation of the predicted output ( $r$ sampling periods ahead) from a desirable output trajectory. Garcia and Morari (1982) have shown that MAC and IMC are very closely related.

It has been shown (Soroush and Kravaris, 1992a) that using nonlinear process models in state-space form and following the conceptual steps of the MAC methodology lead exactly to the GLC error-feedback structure, and application of the GLC 
error-feedback structure to linear systems results in a linear controller, which is identical to the MAC and IMC.

In what follows, we extend our state-space MAC results (Soroush and Kravaris, 1992a) to SISO processes in which $p$ disturbances can be measured on-line. Intuitively, it makes sense to use the available on-line measurements of the disturbances in the output predictions to improve the quality of the predictions. Our focus in this article is on processes with stable finite zero dynamics. The derived nonlinear MAC will turn out to be identical to the controller of Corollary 1 , which was developed in the previous section.

\section{State-space reformulation of $M A C$ for linear processes with measurable disturbances}

Consider linear processes described by a discrete-time statespace model of the form of Eq. 40:

$$
\left\{\begin{aligned}
x_{m}(k+1) & =A x_{m}(k)+b u(k)+\sum_{j=1}^{p} \zeta_{j} d_{j}(k) \\
y_{m}(k) & =c x_{m}(k)
\end{aligned}\right.
$$

where, in the above equations, the subscript $m$ has been added to explicitly indicate that $x_{m}$ and $y_{m}$ represent estimates of $x$ and $y$ calculated by simulating the process model, given the manipulated input move $u(k)$ and the disturbance measurements $d_{1}(k), \ldots, d_{p}(k)$. The measured output will still be denoted by $y$. The future behavior of the process can be predicted by using the identities of Eq. 41 as:

$$
\begin{aligned}
& y_{m}(k+i)=c A^{i} x_{m}(k)+\sum_{j=1}^{p} \sum_{i=0}^{i-\rho_{j}} c A^{i-l-1} \zeta_{j} d_{j}(k+l), \\
& i=1, \ldots, r-1
\end{aligned}
$$$$
y_{m}(k+r)=c A^{r} x_{m}(k)+c A^{r-1} b u(k)
$$$$
+\sum_{j=1}^{p} \sum_{l=0}^{r-\rho_{j}} c A^{r-l-1} \zeta_{j} d_{j}(k+l)
$$

where the state estimates $x_{m}$ are calculated from on-line simulation of the process model (Eq. 53). The above prediction equations imply that, for the exact prediction of the future output values, we need to know the future measurements of the $\mathcal{C}$-type disturbances. In the absence of any model for the disturbances, the best that we can do is to assume the future measurements of the $\mathcal{C}$-type disturbances to be equal to their present measurements. Therefore, we can use the above prediction equations to predict the approximate future changes in the output as follows:

$$
\begin{aligned}
& y_{m}(k+i)-y_{m}(k) \approx\left(c A^{i}-c\right) x_{m}(k)+ \sum_{j=1}^{p} \sum_{l=0}^{i-\rho_{j}} c A^{i-l-1} \zeta_{j} d_{j}(k), \\
& i=1, \ldots, r-1 \\
& y_{m}(k+r)-y_{m}(k) \approx\left(c A^{r}-c\right) x_{m}(k)+c A^{r-1} b u(k) \\
&+ \sum_{j=1}^{p} \sum_{l=0}^{r-\rho_{j}} c A^{r-l-1} \zeta_{j} d_{j}(k)
\end{aligned}
$$

When these approximate predicted changes are added to the measured output signal $y(k)$, one obtains the following "closed-loop" prediction equations:

$$
\begin{aligned}
\hat{y}(k+i) \triangleq y(k)+\left(c A^{i}-c\right) x_{m}(k) & +\sum_{j=1}^{p} \sum_{i=0}^{i-\rho_{j}} c A^{i-1-1} \zeta d_{j}(k), \\
i=1, \ldots, r-1 & \\
\hat{y}(k+r) \triangleq y(k)+\left(c A^{r}-c\right) x_{m}(k) & +c A^{r-1} b u(k) \\
& +\sum_{j=1}^{p} \sum_{i=0}^{r-\rho_{j}} c A^{r-1-1} \zeta d_{j}(k)
\end{aligned}
$$

where the superscript ${ }^{\wedge}$ is used to indicate that $\hat{y}$ represents a prediction of the output.

At every time step, the control computer can calculate the output predictions (Eq. 54), driven by $u(k), d_{\Theta}(k), d_{\mathrm{e}}(k)$ and $y(k)$, where $x_{m}(k)$ is obtained by on-line simulation of the state equations of Eq. 53 , which is driven by the manipulated input and measurements of the measurable disturbances.

In the MAC methodology, the controller move $u(k)$ is calculated by solving the minimization problem:

$$
\min _{u(k)}\left[y_{d}(k+r)-\hat{y}(k+r)\right]^{2}
$$

where $\hat{y}(k+r)$ is defined in Eq. 54 and $y_{d}(k+r)$ is obtained from:

$$
y_{d}(k+r)=(1-\alpha) y_{s p}+\alpha \hat{y}(k+r-1)
$$

with $\alpha$ being a tunable parameter such that $0<\alpha<1$. Equation 56 is referred to as "reference trajectory" in the MAC literature.

The minimization problem of Eq. 55 is trivially solvable leading to the following control law (in the absence of input constraints):

$$
\begin{gathered}
u(k)=-\left(\frac{c A^{r}-\alpha c A^{r-1}-(1-\alpha) c}{c A^{r-1} b}\right) x_{m}(k) \\
+\left(\frac{1-\alpha}{c A^{r-1} b}\right) e(k) \\
-\frac{\sum_{j=1}^{p}\left[\sum_{l=0}^{r-\rho_{j}} c A^{r-l-1}-\alpha \sum_{l=0}^{r-\rho_{j}-1} c A^{r-1-2}\right] \zeta_{j} d_{j}(k)}{c A^{r-1} b}
\end{gathered}
$$

where $x_{m}(k)$ is obtained by simulating:

$$
x_{m}(k+1)=A x_{m}(k)+b u(k)+\sum_{j=1}^{p} \zeta_{j} d_{j}(k)
$$

This controller is identical to the controller realization of Eq. 48 with $x_{m}$ replacing $w$. 


\section{$M A C$ for nonlinear processes with measurable disturbances}

The steps of the state-space linear MAC of the previous subsection can be extended "word by word" to nonlinear processes described by discrete-time models of the form of Eq. $1:$

$$
\left\{\begin{aligned}
x_{m}(k+1) & =\Phi\left[x_{m}(k), u(k), d_{1}(k), \ldots, d_{p}(k)\right] \\
y_{m}(k) & =h\left[x_{m}(k)\right]
\end{aligned}\right.
$$

where again the subscript $m$ is added to indicate estimates of $x$ and $y$ obtained by model simulation and differentiate the calculated $y$ from the measured $y$.

In this case, the approximate future changes in the output $y$ are predicted as follows:

$y_{m}(k+l)-y_{m}(k) \approx h^{\prime}\left[x_{m}(k), d_{\mathfrak{e}}(k)\right]-h\left[x_{m}(k)\right]$,

$$
l=1, \ldots, r-1
$$

$y_{m}(k+r)-y_{m}(k) \approx h^{r-1}\left\{\Phi\left[x_{m}(k), u(k), d_{1}(k), \ldots\right.\right.$,

$$
\left.\left.d_{p}(k)\right], d_{\mathrm{e}}(k)\right]-h\left[x_{m}(k)\right]
$$

where $x_{m}(k)$ is obtained from the on-line simulation of the process model (Eq. 57), and therefore, the "closed-loop" predictions of the output are given by:

$\hat{y}(k+l) \triangleq y(k)+h^{\prime}\left[x_{m}(k), d_{\mathcal{C}}(k)\right]-h\left[x_{m}(k)\right]$, $l=1, \ldots, r-1$

$\hat{y}(k+r) \triangleq y(k)+h^{r-1}\left[\Phi\left(x_{m}(k), u(k), d_{1}(k), \ldots\right.\right.$,

$$
\left.\left.d_{p}(k)\right), d_{\mathrm{e}}(k)\right]-h\left[x_{m}(k)\right]
$$

A nonlinear $\mathrm{MAC}$ can be derived by solving the minimization problem of Eq. 55, where $y_{d}(k+r)$ and $\hat{y}(k+r)$ are calculated from Eqs. 56 and 58, respectively. In the absence of input constraints, the minimizing control move $u(k)$ is the solution of the nonlinear algebraic equation:

$$
\begin{aligned}
h^{r-1}\left\{\Phi\left[x_{m}(k), u(k), d_{1}(k), \ldots, d_{p}(k)\right], d_{\mathrm{C}}(k)\right\} \\
=\alpha h^{r-1}\left[x_{m}(k), d_{\mathrm{C}}(k)\right]+(1-\alpha)\left\{e(k)+h\left[x_{m}(k)\right]\right\}
\end{aligned}
$$

Remembering the definition of $\Psi_{0}$ (Eqs. 21 and 22), the solution can be represented as:

$$
\begin{array}{r}
u(k)=\Psi_{o}\left\{x_{m}(k), d_{\mathbb{B}}(k), d_{\mathrm{e}}(k),(1-\alpha)\left\{e(k)+h\left[x_{m}(k)\right]\right\}\right. \\
\left.+\alpha h^{r-1}\left[x_{m}(k), d_{\mathrm{C}}(k)\right]\right\}
\end{array}
$$

Thus, the derived control law is given by:

$$
\begin{aligned}
u(k)= & \Psi_{o}\left\{x_{m}(k), d_{\mathbb{B}}(k), d_{\mathfrak{C}}(k),\right. \\
& \left.(1-\alpha)\left\{e(k)+h\left[x_{m}(k)\right]\right\}+\alpha h^{r-1}\left[x_{m}(k), d_{\mathfrak{e}}(k)\right]\right\}
\end{aligned}
$$

where $x_{m}(k)$ is obtained by simulating

$x_{m}(k+1)=\Phi\left[x_{m}(k), u(k), d_{1}(k), \ldots, d_{p}(k)\right]$ which is identical to the controller realization of Eq. 38 with $x_{m}$ replacing $w$.

Remark 11. In the derivation of a MAC controller, one can penalize the input by including an additional term in the performance index:

$$
\min _{u(k)}\left\{\left(y_{d}(k+r)-\hat{y}(k+r)\right)^{2}+\mathbb{R}(u(k))^{2}\right\}
$$

where $R$ is a positive tunable parameter. Minimization of such a performance index in the presence of the inputs constraints $u_{l} \leq u(k) \leq u_{h}$ leads to the following general control law:

$u(k)=\delta\left\{\psi\left[x_{m}(k), d_{\mathbb{B}}(k), d_{\mathcal{C}}(k), e(k)\right]\right\}$

where $x_{m}(k)$ is obtained by simulating

$x_{m}(k+1)=\Phi\left[x_{m}(k), u(k), d_{1}(k), \ldots, d_{p}(k)\right]$

Here:

$$
\delta(w) \triangleq \begin{cases}u_{l}, & \text { if } w<u_{l} \\ w, & \text { if } u_{l} \leq w<u_{h} \\ u_{h}, & \text { if } w \geq u_{h}\end{cases}
$$

is the saturation function, and $\psi\left[x_{m}(k), d_{\mathbb{B}}(k), d_{\mathrm{e}}(k), e(k)\right]$ is the corresponding implicit solution for $u$ of the nonlinear algebraic equation:

$$
\begin{gathered}
\frac{\mathbb{R u}(k)}{\frac{\partial h^{r-1}\left[\Phi\left(x_{m}(k), u(k), d_{1}(k), \ldots, d_{p}(k)\right), d_{\mathfrak{C}}(k)\right]}{\partial u(k)}} \\
+h^{r-1}\left[\Phi\left(x_{m}(k), u(k), d_{1}(k), \ldots, d_{p}(k)\right), d_{\mathrm{e}}(k)\right] \\
=\alpha h^{r-1}\left[x_{m}(k), d_{\mathrm{e}}(k)\right]+(1-\alpha)\left\{e(k)+h\left[x_{m}(k)\right]\right\}
\end{gathered}
$$

where $x_{m}(k)$ is obtained by on-line simulation of the process model (Eq. 57). Under nonzero $R$ or an active input constraint, the above control law, even when $\rho_{j} \geq r, j=1, \ldots, p$, does not induce a linear input/output behavior to the closed-loop system and its theoretical properties are unknown at the present time. The controller of Eq. 59 is a special case of the general controller of Eq. 61.

\section{Illustrative Example: Application to a Continuous Stirred-Tank Reactor (CSTR)}

To study the performance of the derived nonlinear control laws, we consider the same reactor example that was used in (Soroush and Kravaris, 1992a). The reactor is a CSTR (shown in Figure 4), in which the parallel reactions:

$$
\begin{aligned}
& A \stackrel{k_{1}}{\rightarrow} U_{1} \\
& A \stackrel{k_{2}}{\rightarrow} U_{2} \\
& A \stackrel{k_{d}}{\rightarrow} D
\end{aligned}
$$




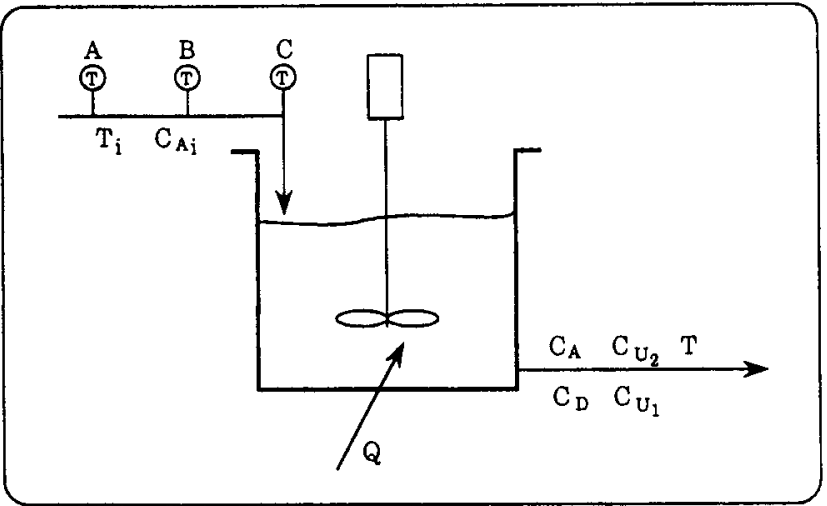

Figure 4. Simulated CSTR.

take place. $U_{1}$ and $U_{2}$ are undesirable side products, and $D$ is the desirable product. It is assumed that the feed to the reactor does not contain $U_{1}, U_{2}$ or $D$. The dependence of the reaction rate constants $k_{1}, k_{2}$ and $k_{d}$ on temperature is given by $k_{i}=Z_{i} \exp \left(-E_{a_{i}} / R T\right), i=1,2$ and $k_{d}=Z_{d} \exp \left(-E_{a_{d}} / R T\right)$.

\section{Mathematical model and control problem}

Energy and species mass balances for the reactor (under standard assumptions) give the reactor model, which is of the form:

$$
\left\{\begin{array}{l}
\frac{d C_{A}}{d t}=R_{A}\left(C_{A}, T\right)+\frac{C_{A_{j}}-C_{A}}{\tau} \\
\frac{d T}{d t}=\frac{R_{H}\left(C_{A}, T\right)}{R, C}+\frac{T_{i}-T}{\tau}+\frac{Q}{R_{r} c V} \\
\frac{d C_{D}}{d t}=R_{D}\left(C_{A}, T\right)-\frac{C_{D}}{\tau}
\end{array}\right.
$$

where the rate expressions $R_{A}\left(C_{A}, T\right), R_{H}\left(C_{A}, T\right)$ and $R_{D}\left(C_{A}\right.$, $T)$ are given by:

$R_{A}\left(C_{A}, T\right)=-k_{1} C_{A}^{n_{1}}-k_{2} C_{A}^{n_{2}}-k_{d} C_{A}^{n_{d}}$

$R_{H}\left(C_{A}, T\right)=\left(-\Delta H_{1}\right) k_{1} C_{A}^{n_{1}}+\left(-\Delta H_{2}\right) k_{2} C_{A}^{n_{2}}+\left(-\Delta H_{d}\right) k_{d} C_{A}^{n_{A}}$

$R_{D}\left(C_{A}, T\right)=k_{d} C_{A}^{n_{A}}$

The control problem is to maintain the reactor temperature $T$ at $400 \mathrm{~K}$, which corresponds to maximum steady-state concentration of the desirable product $\left(C_{D_{s s}}=4.0 \mathrm{kmol} / \mathrm{m}^{3}\right)$, in the presence of process disturbances and modeling errors, by manipulating the heat input to the reactor $(Q)$. As given in Table

Table 1. Steady-State Operating Points Corresponding to $u_{s s}=-1.030 \mathrm{~kJ} \cdot \mathrm{s}^{-1}$

\begin{tabular}{cccc}
\hline $\begin{array}{c}\text { Steady-State } \\
\text { Operating } \\
\text { Point }\end{array}$ & $\begin{array}{c}C_{A_{s s}} \\
\left(\mathrm{kmol} \cdot \mathrm{m}^{-3}\right)\end{array}$ & $\begin{array}{c}C_{D_{s s}} \\
\left(\mathrm{kmol} \cdot \mathrm{m}^{-3}\right)\end{array}$ & $\begin{array}{c}T_{s s} \\
(\mathrm{~K})\end{array}$ \\
\hline SS1 & 7.925 & 0.178 & 310.8 \\
SS2 & 3.321 & 2.520 & 370.0 \\
SS3 & 1.320 & 4.000 & 400.0 \\
\hline
\end{tabular}

1, for $u_{s s}=-1.030 \mathrm{~kJ} \cdot \mathrm{s}^{-1}$ there are three steady-state operating points: SS1, SS2 and SS3. The steady-state operating points SS1 and SS3 are stable, whereas the steady-state operating point SS2 is not stable. Therefore, the control problem is to maintain the reactor at the high conversion, high temperature steady-state operating point SS3, in the presence of disturbances in the inlet temperature $T_{i}$.

For the controller design, since $C_{D}$ does not affect $T$, only the first two differential equations of Eq. 62 are needed. Therefore, the process model, in a vector form, can be written as:

$$
\left\{\begin{array}{c}
\frac{d}{d t}\left[\begin{array}{c}
C_{A} \\
T
\end{array}\right]=\left[\begin{array}{c}
R_{A}\left(C_{A}, T\right)+\frac{C_{A_{i}}-C_{A}}{\tau} \\
\frac{R_{H}\left(C_{A}, T\right)}{R_{r} c}-\frac{T}{\tau}
\end{array}\right] \\
+\left[\begin{array}{c}
0 \\
\frac{1}{R_{r} c V}
\end{array}\right] u+\left[\begin{array}{l}
0 \\
\frac{1}{\tau}
\end{array}\right] d \\
y=T
\end{array}\right.
$$

where $u=Q$ and $d=T_{i}$.

Discrete-Time Model through Forward Difference Approximation. For the discretization of the above continuous-time model, a simple forward difference method (Euler's method) is used, which can provide a good approximate discrete model under fast sampling. The resulting discrete-time model is:

$$
\left\{\begin{aligned}
{\left[\begin{array}{c}
C_{A}(k+1) \\
T(k+1)
\end{array}\right] } & =\left[\begin{array}{c}
F_{1}\left[C_{A}(k), T(k)\right] \\
F_{2}\left[C_{A}(k), T(k)\right]+\frac{\Delta t}{\mathcal{A}_{,} c V} u(k)+\frac{\Delta t}{\tau} d(k)
\end{array}\right] \\
y(k) & =T(k)
\end{aligned}\right.
$$

where $\Delta t$ is the sampling period and

$F_{1}\left[C_{A}(k), T(k)\right] \triangleq C_{A}(k)+R_{A}\left[C_{A}(k), T(k)\right] \Delta t$

$$
+\frac{\Delta t}{\tau}\left[C_{A_{t}}-C_{A}(k)\right]
$$

$F_{2}\left[C_{A}(k), T(k)\right] \triangleq T(k)+\frac{R_{H}\left[C_{A}(k), T(k)\right] \Delta t}{R_{,} c}-\frac{\Delta t}{\tau} T(k)$

The above discrete-time model will be used under a slow sampling rate $(\Delta t=10 \mathrm{~s})$, and as we will see, the nonlinear controller is robust to the modeling errors originating from the use of the very approximate time-discretization method and can induce the theoretically-requested closed-loop response.

To study the performance of the developed nonlinear feedforward/feedback control method in the presence of the three classes of measurable disturbances and compare the performance of the feedforward/feedback controllers to that of a pure 
feedback controller, it is assumed that the heat input enters the reactor with a time-delay equivalent to one sampling period, and the inlet temperature $T_{i}$ can be measured by one of the three sensors located at the positions A, B, and C shown in Figure 4, or cannot be measured. Therefore, we consider the following four cases:

- Case A: The inlet temperature $T_{i}$ is measured by the sensor located at the position A (upstream of the reactor), from where it takes two sampling periods for the inlet stream to reach the reactor. Therefore, in this case, a disturbance detected by the sensor $A$ will enter the process after a time-delay equivalent to two sampling periods.

- Case B: The inlet temperature $T_{i}$ is measured by a sensor located at the position $B$ (upstream of the reactor), from where it takes one sampling period for the inlet stream to reach the reactor. Therefore, in this case, a disturbance detected by the sensor $B$ will enter the process after a time-delay equivalent to one sampling period.

- Case $\mathrm{C}$ : The inlet temperature $T_{i}$ is measured by the sensor located at the position $C$ (upstream of the reactor), exactly where the inlet stream enters the reactor. Therefore, in this case, a disturbance detected by the sensor $C$ will enter the process without time-delay.

- Case D: The inlet temperature $T_{i}$ is not measured, and for controller synthesis, it is assumed to be constant $\left[T_{i}(k)=295.2 \mathrm{~K}\right]$. Therefore, in this case, the feedforward/ feedback control law reduces to a pure feedback controller.

In what follows, using the model of Eq. 63, for each case we obtain a discrete-time model in the form of Eq. 1. These discrete-time models are used to synthesize a nonlinear controller for each case.

Discrete-Time Model of Case A.

$$
\left\{\begin{aligned}
{\left[\begin{array}{c}
C_{A}(k+1) \\
T(k+1) \\
x_{3}(k+1) \\
x_{4}(k+1) \\
x_{5}(k+1)
\end{array}\right] }
\end{aligned}\right]\left[\begin{array}{c}
F_{1}\left[C_{A}(k), T(k)\right] \\
F_{2}\left[C_{A}(k), T(k)\right]+\frac{\Delta t}{\mathbb{R}_{,} V} x_{3}(k)+\frac{\Delta t}{\tau} x_{4}(k) \\
u(k) \\
x_{5}(k) \\
d(k)
\end{array}\right]
$$

Therefore, in this case, the vector of state variables $x=\left[C_{A} T\right.$ $\left.x_{3} x_{4} x_{5}\right]^{T} \in \mathbb{R}^{s}$.

Discrete-Time Model of Case B.

$$
\left\{\begin{aligned}
{\left[\begin{array}{c}
C_{A}(k+1) \\
T(k+1) \\
x_{3}(k+1) \\
x_{4}(k+1)
\end{array}\right] } & =\left[\begin{array}{c}
F_{1}\left[C_{A}(k), T(k)\right] \\
F_{2}\left[C_{A}(k), T(k)\right]+\frac{\Delta t}{R_{1} c V} x_{3}(k)+\frac{\Delta t}{\tau} x_{4}(k) \\
u(k) \\
d(k)
\end{array}\right] \\
y(k) & =T(k)
\end{aligned}\right.
$$

Therefore, in this case, the vector of state variables $x=\left[C_{A} T\right.$ $\left.x_{3} x_{4}\right]^{T} \in \mathbb{R}^{4}$.
Discrete-Time Model of Case C.

$$
\left\{\begin{array}{c}
{\left[\begin{array}{c}
C_{A}(k+1) \\
T(k+1) \\
x_{3}(k+1)
\end{array}\right]=\left[\begin{array}{c}
F_{1}\left[C_{A}(k), T(k)\right] \\
F_{2}\left[C_{A}(k), T(k)\right]+\frac{\Delta t}{R_{1} c V} x_{3}(k)+\frac{\Delta t}{\tau} d(k) \\
u(k)
\end{array}\right]} \\
y(k)=T(k)
\end{array}\right.
$$

Therefore, in this case, the vector of state variables $x=\left[C_{A} T\right.$ $\left.x_{3}\right]^{T} \in \mathbb{R}^{3}$.

Discrete-Time Model of Case D. For this case, the process model is the same as that of Case C (Eq. 66) with $T_{i}=295.2$ $\mathrm{K}$ replacing $d(k)$.

As we saw in the above cases, an increase equivalent to one sampling period in either plant or disturbance time delay, simply increases the order of process model by one.

\section{Controller synthesis and implementation}

The first step is to calculate the relative orders of the process models (Eqs. 64, 65 and 66) according to Definitions 1 and 2.

- Case A: Since

$$
\begin{gathered}
\frac{\partial h(x)}{\partial x} \frac{\partial \Phi(x, u, d)}{\partial u}=0, \\
\frac{\partial h(x)}{\partial x} \frac{\partial \Phi(x, u, d)}{\partial x} \frac{\partial \Phi(x, u, d)}{\partial u}=\frac{\Delta t}{R_{,} c V} \not 0 \\
\frac{\partial h(x)}{\partial x} \frac{\partial \Phi(x, u, d)}{\partial d}=0, \frac{\partial h(x)}{\partial x} \frac{\partial \Phi(x, u, d)}{\partial x} \frac{\partial \Phi(x, u, d)}{\partial d}=0, \\
\frac{\partial h(x)}{\partial x}\left[\frac{\partial \Phi(x, u, d)}{\partial x}\right]^{2} \frac{\partial \Phi(x, u, d)}{\partial d}=\frac{\Delta t}{\tau} \neq 0,
\end{gathered}
$$

and therefore, the relative orders are $r=2$ and $\rho=3$. In this case $\rho>r$; thus, the disturbance $d$ is a Q-type disturbance.

- Case B: Since

$$
\begin{gathered}
\frac{\partial h(x)}{\partial x} \frac{\partial \Phi(x, u, d)}{\partial u}=0 \\
\frac{\partial h(x)}{\partial x} \frac{\partial \Phi(x, u, d)}{\partial x} \frac{\partial \Phi(x, u, d)}{\partial u}=\frac{\Delta t}{R_{r} c V} \neq 0 \\
\frac{\partial h(x)}{\partial x} \frac{\partial \Phi(x, u, d)}{\partial d}=0 \\
\frac{\partial h(x)}{\partial x} \frac{\partial \Phi(x, u, d)}{\partial x} \frac{\partial \Phi(x, u, d)}{\partial d}=\frac{\Delta t}{\tau} \neq 0
\end{gathered}
$$

and therefore, the relative orders are $r=2$ and $\rho=2$. In this case $\rho=r$; thus, the disturbance $d$ is a $B$-type disturbance.

- Case C: Since

$$
\begin{gathered}
\frac{\partial h(x)}{\partial x} \frac{\partial \Phi(x, u, d)}{\partial u}=0, \\
\frac{\partial h(x)}{\partial x} \frac{\partial \Phi(x, u, d)}{\partial x} \frac{\partial \Phi(x, u, d)}{\partial u}=\frac{\Delta t}{R_{r} c V} \neq 0, \\
\frac{\partial h(x)}{\partial x} \frac{\partial \Phi(x, u, d)}{\partial d}=\frac{\Delta t}{\tau} \neq 0,
\end{gathered}
$$


and therefore, the relative orders are $r=2$ and $\rho=1$. In this case $\rho<r$; thus, the disturbance $d$ is a e-type disturbance.

- Case D: In this case, there is no measurable disturbance $(p=0)$, and as in the other cases, the relative order $r=2$.

As was seen in the above cases, the increase of the relative order $\rho$ by one represents one sampling period earlier detection of the disturbance.

The controller of Remark 9 (Eq. 32 in conjunction with a reduced-order open-loop state observer) with $\beta_{1}=\gamma_{1}=-\alpha$ and $\beta_{2}=\gamma_{2}=0$ for the four cases takes the forms:

- Case A:

$$
\begin{array}{r}
u(k)=\left\{\alpha\left[F_{2}\left[w_{1}(k), T(k)\right]+\frac{\Delta t}{\Omega_{r} c V} w_{3}(k)\right]\right. \\
-F_{2}\left(F_{1}\left[w_{1}(k), T(k)\right], F_{2}\left[w_{1}(k), T(k)\right]+\frac{\Delta t}{\mathbb{R}_{r} c V} w_{3}(k)\right. \\
\left.+\frac{\Delta t}{\tau} w_{4}(k)\right)+(1-\alpha)\left[\xi_{2}(k)+e(k)\right] \\
\left.+\frac{\Delta t}{\tau}\left[\alpha w_{4}(k)-w_{5}(k)\right]\right\} \frac{R_{r} c V}{\Delta t}
\end{array}
$$

where $\xi_{2}(k), w_{1}(k), w_{3}(k), w_{4}(k)$, and $w_{5}(k)$ are obtained by simulating:

$$
\begin{array}{ll}
\xi_{1}(k+1)=\alpha \xi_{1}(k) & w_{1}(k+1)=F_{1}\left[w_{1}(k), T(k)\right] \\
+(1-\alpha)\left[\xi_{2}(k)+e(k)\right] & w_{3}(k+1)=u(k) \\
\xi_{2}(k+1)=\xi_{1}(k) & w_{4}(k+1)=w_{5}(k) \\
& w_{5}(k+1)=d(k)
\end{array}
$$

with the nominal initial conditions $\xi_{1}(0)=\xi_{2}(0)=T(0)$, $w_{1}(0)=C_{A}(0), w_{3}(0)=u_{s s}, w_{4}(0)=w_{5}(0)=d(0)$.

- Case B:

$$
\begin{array}{r}
u(k)=\left\{\alpha\left[F_{2}\left[w_{1}(k), T(k)\right]+\frac{\Delta t}{\mathrm{R}_{r} c V} w_{3}(k)\right]\right. \\
-F_{2}\left(F_{1}\left[w_{1}(k), T(k)\right], F_{2}\left[w_{1}(k), T(k)\right]+\frac{\Delta t}{\mathbb{R}_{r} c V} w_{3}(k)\right. \\
\left.+\frac{\Delta t}{\tau} w_{4}(k)\right)+(1-\alpha)\left[\xi_{2}(k)+e(k)\right] \\
\left.+\frac{\Delta t}{\tau}\left[\alpha w_{4}(k)-d(k)\right]\right\} \frac{R_{r} c V}{\Delta t}
\end{array}
$$

where $\xi_{2}(k), w_{1}(k), w_{3}(k)$, and $w_{4}(k)$ are obtained by simulating:

$$
\begin{array}{ll}
\xi_{1}(k+1)=\alpha \xi_{1}(k) & w_{1}(k+1)=F_{1}\left[w_{1}(k), T(k)\right] \\
+(1-\alpha)\left[\xi_{2}(k)+e(k)\right] & w_{3}(k+1)=u(k) \\
\xi_{2}(k+1)=\xi_{1}(k) & w_{4}(k+1)=d(k)
\end{array}
$$

with the nominal initial conditions $\xi_{1}(0)=\xi_{2}(0)=T(0)$, $w_{1}(0)=C_{A}(0), w_{3}(0)=u_{s s}, w_{4}(0)=d(0)$.

- Case C:

$$
\begin{aligned}
& u(k)=\left\{\alpha\left[F_{2}\left[w_{1}(k), T(k)\right]+\frac{\Delta t}{R_{r} c V} w_{3}(k)\right]\right. \\
& -F_{2}\left(F_{1}\left[w_{1}(k), T(k)\right], F_{2}\left[w_{1}(k), T(k)\right]+\frac{\Delta t}{R_{r} c V} w_{3}(k)\right. \\
& \left.\left.+\frac{\Delta t}{\tau} d(k)\right)+(1-\alpha)\left[\xi_{2}(k)+e(k)-\frac{\Delta t}{\tau} d(k)\right]\right\} \frac{R_{,} c V}{\Delta t} \\
& \text { where } \xi_{2}(k), w_{1}(k), \text { and } w_{3}(k) \text { are obtained by simulating: } \\
& \xi_{1}(k+1)=\alpha \xi_{1}(k)+(1-\alpha)\left[\xi_{2}(k)+e(k)\right] \\
& \xi_{2}(k+1)=\xi_{1}(k) \\
& w_{1}(k+1)=F_{1}\left[w_{1}(k), T(k)\right] \\
& w_{3}(k+1)=u(k)
\end{aligned}
$$

with the nominal initial conditions $\xi_{1}(0)=\xi_{2}(0)=T(0)$, $w_{1}(0)=C_{A}(0), w_{3}(0)=u_{s s}$.

- Case D: The controller is the same as that of Case $C(\mathrm{Eq}$. 69) with $T_{i}=295.2 \mathrm{~K}$ replacing $d(k)$.

In the absence of modeling errors and unmeasurable disturbances, the controllers of Eqs. 67 and 68 completely elim-

\begin{tabular}{|c|c|c|}
\hline $\begin{array}{l}T(0)=T_{s s} \\
C_{A}(0)=C_{A_{s s}}\end{array}$ & $\begin{array}{l}=4.00 \times 10^{2} \\
=1.32 \times 10^{0}\end{array}$ & $\begin{array}{l}\mathrm{K} \\
\mathrm{kmol} \cdot \mathrm{m}^{-3}\end{array}$ \\
\hline$C_{D}(0)=C_{D_{s s}}$ & $=4.00 \times 10^{0}$ & $\mathrm{kmol} \cdot \mathrm{m}^{-3}$ \\
\hline$Q_{s s}$ & $=-1.03 \times 10^{0}$ & $\mathrm{~kJ} \cdot \mathrm{s}^{-1}$ \\
\hline
\end{tabular}
inate the measurable disturbance and induce the linear input/ output behavior (first-order-plus-deadtime response):

$$
y(k+2)-\alpha y(k+1)=(1-\alpha) y_{s p}(k)
$$

to the closed-loop process. The above controllers include reduced-order state observers, which are used to calculate the estimates of all the states except the reactor temperature.

\section{Simulation results}

To simulate the reactor process, the standard software package ODEPACK is used to integrate numerically the ordinary differential equations in Eq. 62. Every $10 \mathrm{~s}$ (the sampling period $\Delta t$ ), the value of the reactor temperature $T$ (calculated by the ODE solver) and the inlet temperature measurement signal $(d)$ are used in the discrete-time controllers as on-line process measurements. The only tunable parameter of the three controllers $(\alpha)$ is chosen to be $\alpha=0.85$.

Using the operating conditions given in Table 2, numerical simulations are performed to examine the regulatory performance of the four controllers of Eqs. 67, 68, 69 and Case D, under no modeling errors (nominal case) and $20 \%$ error in the reactor residence time. In particular, the objective is to inves-

Table 2. Operating Conditions of the CSTR 
tigate the ability of the four controllers in rejecting the effect of a step change ( $30 \mathrm{~K}$ increase) in the inlet temperature $T_{i}$.

Figure $5 \mathrm{a}$ depicts the regulatory performance of the controllers in rejecting the effect of a step disturbance in $T_{i}(30$ deg. increase, from $22^{\circ} \mathrm{C}$ to $52^{\circ} \mathrm{C}$, at $t=100 \mathrm{~s}$ ). As this figure shows, the effect of the disturbance on the output is completely eliminated in Cases A and B. However, in Case C, the process evolves in an open-loop manner for one sampling period, after which the controller action asymptotically brings the output back to its set point. The simulation results shown in Figure $5 \mathrm{a}$ are in agreement with the theoretically-requested closedloop response (Eq. 70), that is, the controllers of Cases $A$ and $B$ can completely eliminate the effect of the disturbance on the output. Note that the complete disturbance elimination shown in Figure $5 \mathrm{a}$ has been achieved in the presence of the modeling errors, which have originated from the use of the simple forward difference approximation under slow sampling. The corresponding manipulated input profiles are depicted in Figure $5 \mathrm{~b}$. As shown in Figure $5 \mathrm{a}$, for one sampling period beyond $t=100 \mathrm{~s}$, the output responses in Cases $C$ and $D$ are

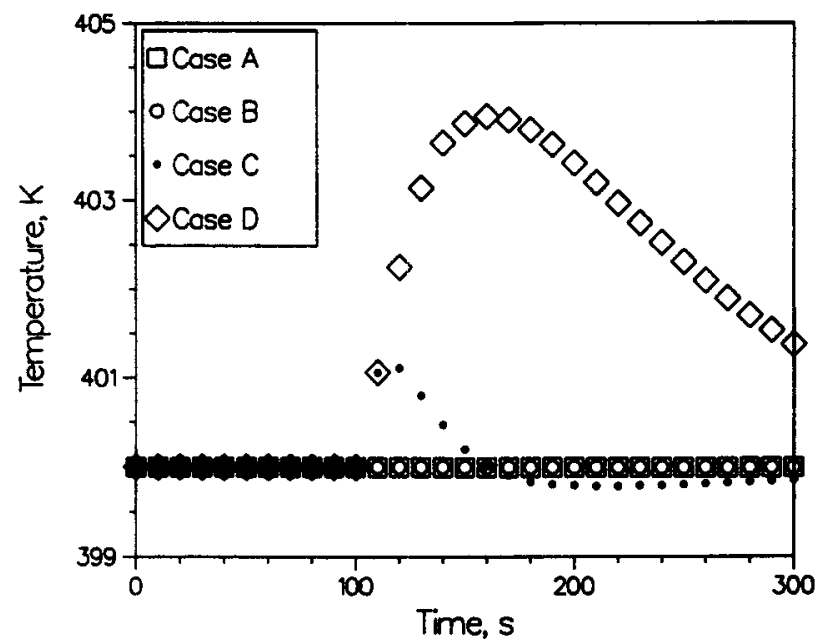

Figure 5a. Closed-loop temperature responses to a step increase in $T_{1}$ at $t=100 \mathrm{~s}$.

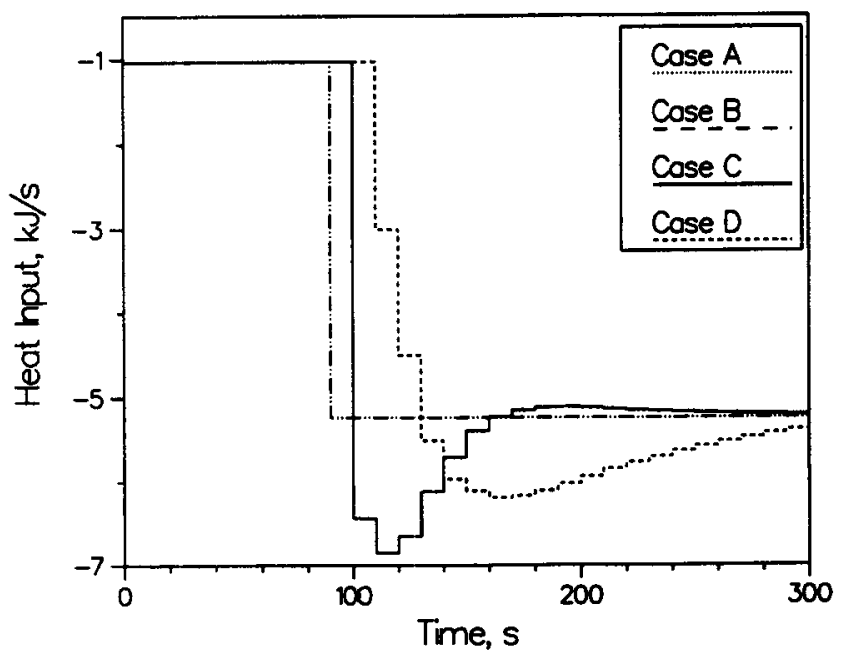

Figure 5b. Profiles of manipulated input corresponding to Figure 5 a. the same. After one sampling period, the controller of Case $\mathrm{D}$ acts, but not as aggressively as those of Cases $\mathrm{A}, \mathrm{B}$ and $\mathrm{C}$, to bring back the reactor temperature to its set point: in Case $\mathrm{D}$, the reactor temperature is strongly affected by the disturbance (initially increases and then returns to its set point after more than $600 \mathrm{~s}$ ). Figure $5 \mathrm{~b}$ also shows that the actions taken by the controllers of Cases A and B are the same. Therefore, the controller of Case $A$ does not act immediately once the disturbance is detected by the sensor $A$; it acts one sampling period later to compensate for the disturbance.

The above simulation results are in complete agreement with our intuition and understanding from linear control theory that an earlier detection of measurable process disturbances results in a more effective compensation for the disturbances. In particular, for the complete elimination of measurable disturbances, we need to detect process disturbances early enough to have the disturbance deadtimes $\left(\tau_{d_{1}}, \ldots, \tau_{d_{p}}\right)$ larger than or equal to the plant deadtime $\left(\tau_{p}\right)$.

Robustness of the feedforward/feedback controller to mod-

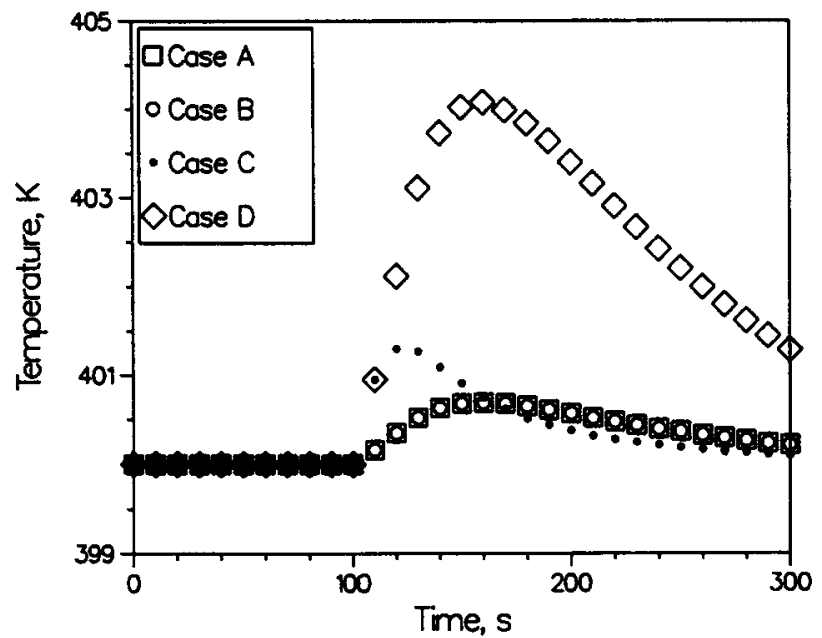

Figure 6a. Closed-loop temperature responses to a step increase in $T_{1}$ at $t=100 \mathrm{~s}$ (under modeling errors).

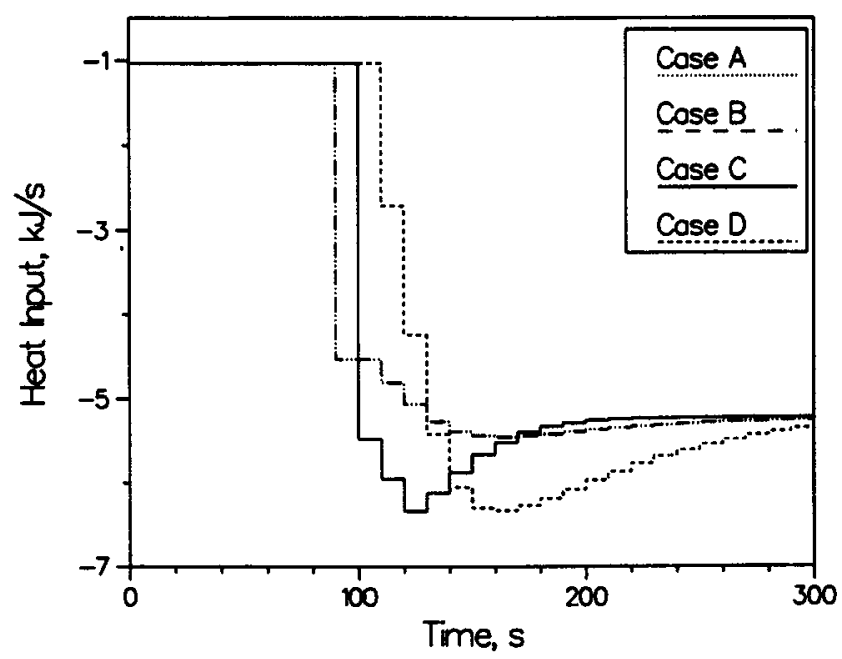

Figure 6b. Profiles of manipulated input corresponding to Figure 6 a. 
eling errors is evaluated by closed-loop simulations under $20 \%$ error in the reactor residence time $(\tau)$. The resulting output responses are shown in Figure 6a. The corresponding manipulated input profiles are depicted in Figure $6 \mathrm{~b}$. In the presence of the modeling error, none of the controllers can completely eliminate the effect of the disturbance on the output; the theoretically requested closed-loop response of Eq. 70 cannot be induced. The closed-loop temperature responses under the controllers of Cases A and B are identical (the actions taken by the controllers of Cases A and B are the same). In Case C, the effect of the disturbance on the temperature is stronger than in Cases A and B. In Case D, the reactor temperature, as in the absence of modeling errors, is strongly affected by the disturbance and returns to its set point after more than $600 \mathrm{~s}$. As expected, even in the presence of modeling errors, the regulatory performance of feedforward/feedback control is superior to that of pure feedback control.

\section{Acknowledgment}

Financial support from the National Science Foundation through the grant CTS-8912836 is gratefully acknowledged.

\section{Notation}

$A=$ reactant

$c=$ heat capacity of reacting mixture, $\mathrm{kJ} \cdot \mathrm{kg}^{-1} \cdot \mathrm{K}^{-1}$

$C_{A}=$ concentration of reactant $A, \mathrm{kmol} \cdot \mathrm{m}^{-3}$

$C_{A_{s s}}=$ steady-state concentration of reactant $\mathrm{A}, \mathrm{kmol} \cdot \mathrm{m}^{-3}$

$C_{A_{i}}=$ inlet concentration of reactant $\mathrm{A}, \mathrm{kmol} \cdot \mathrm{m}^{-3}$

$C_{D}=$ concentration of desirable product, $\mathrm{kmol} \cdot \mathrm{m}^{-3}$

$D_{D_{s s}}=$ steady-state concentration of desirable product, $\mathrm{kmol} \cdot \mathrm{m}^{-3}$

$C_{U_{l}}=$ concentration of undesirable product $U_{l}, \mathrm{kmol} \cdot \mathrm{m}^{-3}$

$d=$ vector of disturbance inputs

$d_{j}=j$ th disturbance input

$D=$ desirable product

$E_{a_{d}}=$ activation energy for desirable reaction, $\mathrm{kJ} \cdot \mathrm{kmol}^{-1}$

$E_{a_{1}}=$ activation energy for undesirable reaction $l, \mathrm{~kJ} \cdot \mathrm{kmol}^{-1}$

$I=n \times n$ identity matrix

$k_{d}=$ reaction rate constant of desirable reaction, $\mathrm{s}^{-1}$

$k_{1}=$ reaction rate constant of reaction $1, \mathrm{~m}^{6} \cdot \mathrm{kmol}^{-2} \cdot \mathrm{s}^{-1}$

$k_{2}=$ reaction rate constant of reaction $2, \mathrm{kmol}^{0.5} \cdot \mathrm{m}^{-1.5} \cdot \mathrm{s}^{-1}$

$n_{d}=$ order of the desirable reaction

$n_{t}=$ order of the undesirable reaction $l$

$Q=$ rate of heat input to reactor, $\mathrm{kJ} \cdot \mathrm{s}^{-1}$

$Q_{s s}=$ steady-state rate of heat input to reactor, $\mathrm{kJ} \cdot \mathrm{s}^{-1}$

$r=$ relative order of controlled output $y$ with respect to $u$

$R=$ universal gas constant, $\mathrm{kJ} \cdot \mathrm{kmol}^{-1} \cdot \mathrm{K}^{-1}$

$\mathcal{R}=$ penalty on controller action

$\mathcal{R}_{r}=$ density of reacting mixture, $\mathrm{kg} \cdot \mathrm{m}^{-3}$

$R_{A}, R_{D}=$ rate of production of $A$ and $D$, respectively, $\mathrm{kmol} \cdot \mathrm{m}^{-3} \cdot \mathrm{s}^{-1}$

$\boldsymbol{R}_{H}=$ overall rate of heat production by chemical reactions, $\mathbf{k J} \cdot \mathrm{kmol}^{-1}$

$t=$ time, $\mathrm{s}$

$T=$ reactor temperature, $\mathrm{K}$

$T_{i}=$ temperature of inlet stream, $\mathrm{K}$

$T_{s s}=$ steady-state reactor temperature, $\mathrm{K}$

$u=$ manipulated input

$U_{1}, U_{2}=$ undesirable products

$V=$ volume of the reacting mixture, $\mathrm{m}^{3}$

$x=$ vector of state variables

$y=$ output variable

$y_{d}=$ reference trajectory

$y_{s p}=$ output set point

$Z_{d}=$ frequency factor for desirable reaction, $\mathrm{s}^{-1}$

$Z_{1}=$ frequency factor for reaction $1, \mathrm{~m}^{6} \cdot \mathrm{kmol}^{-2} \cdot \mathrm{s}^{-1}$

$Z_{2}=$ frequency factor for reaction $2, \mathrm{kmol}^{0.5} \cdot \mathrm{m}^{-1.5} \cdot \mathrm{s}^{-1}$

\section{Greek letters}

$\beta_{j}=$ tunable parameters of inner loop

$\gamma_{j}=$ tunable parameters of overall closed-loop system

$-\Delta H_{d}=$ heat of desirable reaction, $\mathrm{kJ} \cdot \mathrm{kmol}^{-1}$

$-\Delta H_{l}=$ heat of undesirable reaction $l, \mathrm{~kJ} \cdot \mathrm{kmol}^{-1}$

$\Delta t=$ sampling period, $\mathrm{s}$

$\rho_{j}=$ relative order of controlled output $y$ with respect to the disturbance $d_{j}$

$\tau=$ CSTR residence time

$\tau_{p}=$ plant deadtime

$\tau_{d_{f}}=$ disturbance deadtime

$\Phi=$ state vector function

$\Psi=$ feedforward/state feedback law

\section{Math symbols}

$$
\begin{aligned}
\triangleq & =\text { is defined } \\
\epsilon & =\text { belongs to } \\
\mathbb{R} & =\text { real line } \\
0 & =\text { composition of functions }
\end{aligned}
$$

\section{Literature Cited}

Biegler, L. T., and J. B. Rawlings, "Optimization Approaches to Nonlinear Model Predictive Control," CPC IV, 543 (1991).

Calvet, J-P., and Y. Arkun, "Feedforward and Feedback Linearization of Nonlinear Systems with Disturbances," Int. J. Contr., 48, 1551 (1988a).

Calvet, J-P., and Y. Arkun, "Feedforward and Feedback Linearization of Nonlinear Systems with Disturbances and its Implementation Using Internal Model Control,"' Ind. Eng. Chem. Res., 27, 1882 (1988b).

Cutler, C. R., and B. L. Ramaker, "Dynamic Matrix Control-A Computer Control Algorithm," AIChE National Meeting, Houston, TX (1979).

Daoutidis, P., and C. Kravaris, "Synthesis of Feedforward/StateFeedback Controllers for Nonlinear Processes," AIChE J., 35, 1602 (1989).

Daoutidis, P., M. Soroush, and C. Kravaris, "Feedforward/Feedback Control of Multivariable Nonlinear Processes," AIChE J., 36, 1471 (1990).

Economou, C. G., M. Morari, and B. O. Palsson, "Internal Model Control. 5. Extension to Nonlinear Systems," Ind. Eng. Process Des. Dev., 25, 403 (1986).

Garcia, C. E., and M. Morari, "Internal Model Control: 1. A Unifying Review and Some New Results," Ind. Eng. Chem. Process Des. Dev., 21, 308 (1982).

Garcia, C. E., and M. Morari, "Internal Model Control. 2. Design Procedure for Multivariable Systems," Ind. Eng. Chem. Res., 24, 472 (1985).

Henson, M. A., and D. E. Seborg, "Theoretical Analysis of Unconstrained Nonlinear Model Predictive Control," Paper No. 145a, AIChE Annual Meeting, Los Angeles, CA (1991).

Hidalgo, P. M., and C. B. Brosilow, "Nonlinear Model Predictive Control of Styrene Polymerization at Unstable Operating Points," Comp. Chem. Eng., 14, 481 (1990).

Isermann, R., Digital Control Systems, 2nd ed., Vol. 1, SpringerVerlag, New York, p. 68 (1989).

Isidori, A., Nonlinear Control Systems: An Introduction, 2nd ed., Springer-Verlag, New York, p. 156 (1989).

Li, W. C., L. T. Biegler, C. G. Economou, and M. Morari, "A Constrained Pseudo-Newton Control Strategy for Nonlinear Systems," Comp. Chem. Eng., 14, 451 (1990).

Kravaris, C., and C. B. Chung, "Nonlinear State Feedback Synthesis by Global Input/output Linearization," AlChE J., 33, 592 (1987).

Mehra, R. K., and R. Rouhani, "Theoretical Considerations on Model Algorithmic Control for Nonminimum Phase Systems," Proc. of $A C C$, TA8-B (1980).

Monaco, S., and D. Normand-Cyrot, "Zero Dynamics of Sampled Nonlinear Systems,"' Sys. \& Cont. Lett., 11, 229 (1988).

Morari, M., and E. Zafiriou, Robust Process Control, Prentice Hall, Englewood Cliffs, NJ (1989). 
Nijmeijer, H., and A. J. van der Schaft, Nonlinear Dynamical Control Systems, Springer-Verlag, New York, p. 437 (1990).

Palmor, Z. J., and D. V. Powers, "Feedforward Properties of Deadtime Compensation Controllers," Proc. of ACC (1981).

Pathwardhan, A. A., J. B. Rawlings, and T. F. Edgar, "Nonlinear Model Predictive Control," Chem. Eng. Comm., 87, 123 (1990).

Prett, D. M., and R. D. Gillette, "Optimization and Constrained Multivariable Control of a Catalytic Cracking Unit," AIChE National Meeting, Houston, Texas (1979).

Richalet, J., A. Rault, J. L. Testud, and J. Papon, "Model Predictive Heuristic Control: Application to Industrial Processes," Automatica, 14, 413 (1978).

Shinskey, F. G., Process Control Systems, McGraw-Hill, New York, 3rd ed. (1988).

Sistu, P. B., and B. W. Bequette, "Nonlinear Predictive Control of Uncertain Processes: Application to a CSTR," AIChE J., 37, 1711 (1991).

Soroush, M., and C. Kravaris, "Discrete-Time Nonlinear Controller Synthesis by Input/Output Linearization," AIChE J., 38, 1923 (1992a).

Soroush, M., and C. Kravaris, "A Continuous-Time Formulation of Nonlinear Model Predictive Control," Proc. of ACC, p. 1561 (1992b).

\section{Appendix: Proofs}

\section{Proof of Theorem 1}

The proof makes use of the definitions of relative orders and the implicit function theorem as follows. The system:

$$
\left\{\begin{aligned}
x(k+1) & =\Phi\left[x(k), u(k), d_{1}(k), \ldots, d_{p}(k)\right] \\
y(k) & =h[x(k)]
\end{aligned}\right.
$$

has relative orders $\rho_{1}, \ldots, \rho_{p}$ and $r$, respectively, with respect to $d_{1}, \ldots, d_{p}$ and $u$, therefore, according to Definitions 1 and 2 , the following relations:

$$
\begin{gathered}
{\left[\frac{\partial h}{\partial x}\right]\left[\frac{\partial \Phi}{\partial x}\right]^{l-1}\left[\frac{\partial \Phi}{\partial u}\right] \equiv 0, \quad l=1, \ldots, r-1,} \\
{\left[\frac{\partial h}{\partial x}\right]\left[\frac{\partial \Phi}{\partial x}\right]^{r-1}\left[\frac{\partial \Phi}{\partial u}\right] \equiv 0} \\
{\left[\frac{\partial h}{\partial x}\right]\left[\frac{\partial \Phi}{\partial x}\right]^{l-1}\left[\frac{\partial \Phi}{\partial d_{j}}\right] \equiv 0, \quad l=1, \ldots, \rho_{j}-1, \quad j=1, \ldots, p} \\
{\left[\frac{\partial h}{\partial x}\right]\left[\frac{\partial \Phi}{\partial x}\right]^{\rho_{j}-1}\left[\frac{\partial \Phi}{\partial d_{j}}\right] \not 0, \quad j=1, \ldots, p}
\end{gathered}
$$

where $\Phi^{\prime}\left[x(k), d_{1}(k), \ldots, d_{p}(k), v(k)\right] \triangleq \Phi(x(k), \Psi[x(k)$, $\left.\left.d_{1}(k), \ldots, d_{p}(k), v(k)\right], d_{1}(k), \ldots, d_{p}(k)\right\}$, with respect to $v, d_{1}, \ldots, d_{p}$, respectively. For the above closed-loop system, it is straightforward to show that:

$\frac{\partial h}{\partial x}\left[\frac{\partial \Phi^{\prime}}{\partial x}\right]^{l-1} \frac{\partial \Phi^{\prime}}{\partial v}=\frac{\partial h}{\partial x}\left[\frac{\partial \Phi}{\partial x}+\frac{\partial \Phi}{\partial u} \frac{\partial \Psi}{\partial x}\right]^{1-1} \frac{\partial \Phi}{\partial u} \frac{\partial \Psi}{\partial v} \equiv 0$, $l=1, \ldots, r-1$,

and

$$
\frac{\partial h}{\partial x}\left[\frac{\partial \Phi^{\prime}}{\partial x}\right]^{r-1} \frac{\partial \Phi^{\prime}}{\partial v}=\left[\frac{\partial h}{\partial x}\right]\left[\frac{\partial \Phi}{\partial x}+\frac{\partial \Phi}{\partial u} \frac{\partial \Psi}{\partial x}\right]^{r-1} \frac{\partial \Phi}{\partial u} \frac{\partial \Psi}{\partial v} \not \equiv 0
$$

therefore, $r^{\prime}=r$. Note that

$$
\frac{\partial \Phi}{\partial x}+\frac{\partial \Phi}{\partial u} \frac{\partial \Psi}{\partial x} \not 0
$$

for a well-defined control problem, otherwise the system of Eq. A1 will not be state controllable. Also,

$$
\frac{\partial \Psi}{\partial v} \not \equiv 0,
$$

according to the condition of Eq. 4. Furthermore, for each disturbance $d_{j}$, we can write:

$$
\begin{aligned}
\frac{\partial h}{\partial x} & {\left[\frac{\partial \Phi^{\prime}}{\partial x}\right]^{l-1} \frac{\partial \Phi^{\prime}}{\partial d_{j}}=\left[\frac{\partial h}{\partial x}\right]\left[\frac{\partial \Phi}{\partial x}+\frac{\partial \Phi}{\partial u} \frac{\partial \Psi}{\partial x}\right]^{l-1}\left[\frac{\partial \Phi}{\partial d_{j}}+\frac{\partial \Phi}{\partial u} \frac{\partial \Psi}{\partial d_{j}}\right], } \\
& =\frac{\partial h}{\partial x}\left[\frac{\partial \Phi}{\partial x}+\frac{\partial \Phi}{\partial u} \frac{\partial \Psi}{\partial x}\right]^{l-1} \frac{\partial \Phi}{\partial d_{j}}+\frac{\partial h}{\partial x}\left[\frac{\partial \Phi}{\partial x}+\frac{\partial \Phi}{\partial u} \frac{\partial \Psi}{\partial x}\right]^{l-1} \frac{\partial \Phi}{\partial u} \frac{\partial \Psi}{\partial d_{j}},
\end{aligned}
$$

$l=1, \ldots, \rho_{j}-1$. Using the above identities, for each disturbance $d_{j}$, we can write:

$$
\frac{\partial h}{\partial x}\left[\frac{\partial \Phi^{\prime}}{\partial x}\right]^{l-1} \frac{\partial \Phi^{\prime}}{\partial d_{j}} \equiv \begin{cases}0, & l=1, \ldots, \rho_{j}-1, \rho_{j}<r \\ 0, & l=1, \ldots, r-1, \rho_{j} \geq r\end{cases}
$$

and

$$
\frac{\partial h}{\partial x}\left[\frac{\partial \Phi^{\prime}}{\partial x}\right]^{\rho_{j}-1} \frac{\partial \Phi^{\prime}}{\partial d_{j}}= \begin{cases}\frac{\partial h}{\partial x}\left[\frac{\partial \Phi}{\partial x}+\frac{\partial \Phi}{\partial u} \frac{\partial \Psi}{\partial x}\right]^{\rho_{j}-1} \frac{\partial \Phi}{\partial d_{j}} \equiv 0, & \rho_{j}<r \\ \frac{\partial h}{\partial x}\left[\frac{\partial \Phi}{\partial x}+\frac{\partial \Phi}{\partial u} \frac{\partial \Psi}{\partial x}\right]^{\rho_{j}-1} \frac{\partial \Phi}{\partial d_{j}}+\frac{\partial h}{\partial x}\left[\frac{\partial \Phi}{\partial x}+\frac{\partial \Phi}{\partial u} \frac{\partial \Psi}{\partial x}\right]^{\rho_{j}-1} \frac{\partial \Phi}{\partial u} \frac{\partial \Psi}{\partial d_{j}} \not \equiv \mathrm{Or} \equiv 0, & \rho_{j} \geq r\end{cases}
$$

hold. Now let $r^{\prime}, \rho_{1}^{\prime}, \ldots, \rho_{p}^{\prime}$ be relative orders of the output of the closed-loop system:

$$
\left\{\begin{aligned}
x(k+1) & =\Phi^{\prime}\left[x(k), d_{1}(k), \ldots, d_{p}(k), v(k)\right] \\
y(k) & =h[x(k)]
\end{aligned}\right.
$$

Therefore, for each disturbance $d_{j}, \rho_{j}^{\prime}=\rho_{j}$, if $\rho_{j}<r$, and $\rho_{j}^{\prime} \geq r$, if $\rho_{j} \geq r$. Q.E.D.

\section{Proof of Theorem 2}

(a) Necessity part: The condition $\rho_{1} \geq r$ is necessary as a 
consequence of Theorem 1: if $\rho_{1}<r, \rho_{1}$ will be preserved in closed-loop and therefore, $y$ cannot be made independent of $d_{1}$. In other words, in this case the disturbance $d_{1}$ affects the output before the controller action tries to compensate for the effect of the disturbance.

(b) Sufficiency part: The sufficiency of $\rho_{1} \geq r$ is established through the derived feedforward/feedback law as follows. For $\rho_{1} \geq r$, the output predictions up to $r$ sampling periods ahead are given by:

$$
\begin{aligned}
& y(k+l)=h^{\prime}[x(k)], \quad l=0, \ldots, r-1 \\
& y(k+r)=h^{r-1}\left\{\Phi\left[x(k), u(k), d_{1}(k)\right]\right\}
\end{aligned}
$$

Since the output predictions $y(k), \ldots, y(k+r)$ do not depend on future values of the measured disturbance $d_{1}$, one can synthesize a state feedback that induces a linear closed-loop response to the process under consideration and completely eliminates the effect of the disturbance on the output $y$.

Finally, verification of the closed-loop dynamics (Eq. 17) induced by the feedback law (Eq. 16) is straightforward and as follows. Under the feedforward/feedback law of Eq. 16, the closed-loop system becomes:

$$
\left\{\begin{array}{c}
x(k+1)=\Phi\left(x(k), \Psi_{o}\left(x(k), d_{1}(k),\left(1+\sum_{i=1}^{r} \beta_{l}\right) v(k)\right.\right. \\
\left.\left.-\sum_{i=1}^{r} \beta_{i} h^{r-1}[x(k)]\right), d_{1}(k)\right) \\
y(k)=h(x(k))
\end{array}\right.
$$

For the above closed-loop system, we can write:

$$
\begin{aligned}
y(k+l)=h^{\prime}[x(k)], \quad l= & 0, \ldots, r-1 \\
y(k+r)=h^{r-1}(\Phi(x(k), & \Psi_{o}\left(x(k), d_{1}(k),\left(1+\sum_{l=1}^{r} \beta_{l}\right) v(k)\right. \\
& \left.\left.\left.\quad-\sum_{l=1}^{r} \beta_{l} h^{r-l}[x(k)]\right), d_{1}(k)\right)\right) \quad(\mathrm{A} 4)
\end{aligned}
$$

Using the definition of $\Psi_{o}$ (Eqs. 12 and 13), the righthand side of the last equation in Eq. A4:

$$
\begin{aligned}
h^{r-1}(\Phi(x(k), & \Psi_{\circ}\left(x(k), d_{1}(k),\left(1+\sum_{l=1}^{r} \beta_{l}\right) v(k)\right. \\
\left.\left.\left.-\sum_{l=1}^{r} \beta_{l} h^{r-l}[x(k)]\right), d_{l}(k)\right)\right) & \\
& =\left(1+\sum_{i=1}^{r} \beta_{l}\right) v(k)-\sum_{i=1}^{r} \beta_{l} h^{r-l}[x(k)]
\end{aligned}
$$

therefore,

$$
y(k+r)=\left(1+\sum_{l=1}^{r} \beta_{l}\right) v(k)-\sum_{l=1}^{r} \beta_{l} h^{r-l}[x(k)]
$$

which is equivalent to

$$
y(k+r)+\sum_{l=1}^{r} \beta_{l} y(k+r-l)=\left(1+\sum_{l=1}^{r} \beta_{l}\right) v(k)
$$

Q.E.D.

\section{Proof of Theorem 3}

Under the feedforward/feedback law of Eq. 18 with $\beta_{1}=\ldots=\beta_{r}=0$, the closed-loop system becomes:

$$
\left\{\begin{aligned}
x(k+1) & =\Phi\left\{x(k), \Psi_{o}\left[x(k), d_{1}(k), v(k)\right], d_{1}(k)\right\} \\
y(k) & =h[x(k)]
\end{aligned}\right.
$$

(a) Consider the above closed-loop system and make a step change in $d_{1}$ at time instant $k$, keep $v$ unchanged $\left[v(k+l)=v_{o}\right.$, $l=0,1, \ldots]$, and assume at time instant $k$ the closed-loop system is at steady-state, that is, $x(k+1)=x(k)$ and $y(k)=v_{0}$. For $\rho_{1}<r$, the closed-loop output predictions up to $r$ sampling periods ahead are given by:

$y(k+l)=h^{\prime}[x(k)]=v_{o}, \quad l=0, \ldots, \rho_{1}-1$

$y(k+l)=h^{\prime}\left[x(k), d_{1}(k)\right] \neq v_{o}, \quad l=\rho_{1}, \ldots, r-1$

$y(k+r)=h^{r-1}\left\{\Phi\left[x(k), \Psi_{o}\left[x(k), d_{1}(k), v_{o}\right], d_{1}(k)\right], d_{1}(k)\right\}$

Using the definition of $\Psi_{0}$ (Eqs. 14 and 15), the righthand side of the last equation in Eq. A5:

$$
h^{r-1}\left\{\Phi\left[x(k), \Psi_{o}\left[x(k), d_{1}(k), v_{o}\right], d_{1}(k)\right], d_{1}(k)\right\}=v_{0}
$$

therefore:

$$
y(k+r)=v_{o}
$$

which means that the output remains at $v_{o}$ during the first $\left(\rho_{1}-1\right)$ steps, deviates from $v_{o}$ during the time period between the time instants $\left(k+\rho_{1}\right)$ and $(k+r-1)$ and finally returns to $v_{o}$ at $r$ th time step and stays there.

(b) Consider a step change in $v$ at time instant $k$ from $v_{o}$ to $v_{1}$, while $d_{1}$ remains unchanged $\left[d_{1}(k+l)=d_{1_{0}}, l=0,1, \ldots\right]$, and assume at time instant $k$ the closed-loop system is at steadystate, that is, $x(k+1)=x(k)$ and $y(k)=v_{o}$. For $\rho_{1}<r$, the closed-loop output predictions up to $r$ sampling periods ahead are given by:

$$
\begin{aligned}
& y(k+l)=h^{\prime}[x(k)]=v_{o}, \quad l=0, \ldots, \rho_{1}-1 \\
& y(k+l)=h^{l}\left[x(k), d_{1_{0}}\right]=v_{o}, \quad l=\rho_{1}, \ldots, r-1 \\
& y(k+r)=h^{r-1}\left\{\Phi\left[x(k), \Psi_{o}\left[x(k), d_{1_{o}}, v_{1}\right], d_{1_{o}}\right], d_{1_{o}}\right\}
\end{aligned}
$$

Using the definition of $\Psi_{o}$ (Eqs. 14 and 15), the righthand side of the last equation in Eq. A6:

$$
h^{r-1}\left\{\Phi\left[x(k), \Psi_{o}\left[x(k), d_{1_{o}}, v_{1}\right], d_{1_{o}}\right], d_{1_{o}}\right\}=v_{1}
$$

therefore,

$$
y(k+r)=v_{1}
$$


which means that the output remains at the old $v\left(v_{o}\right)$ during the first $(r-1)$ sampling periods and reaches the new value $v_{1}$ at the $r$ th time instant and stays there (deadbeat control). Q.E.D.

\section{Proof of Theorem 4}

Part $a$. For processes with $\rho_{j} \geq r, j=1, \ldots, p$, under the feedforward/feedback law of Eq. 24, the closed-loop system becomes:

$$
\left\{\begin{aligned}
& x(k+1)=\Phi\left(x(k), \Psi_{o}\left(x(k), d_{\mathbb{B}}(k),\left(1+\sum_{l=1}^{r} \beta_{l}\right) v(k)\right.\right. \\
&\left.\left.-\sum_{l=1}^{r} \beta_{l} h^{r-1}[x(k)]\right), d_{\mathbb{Q}}(k), d_{\mathbb{B}}(k)\right) \\
& y(k)=h(x(k))
\end{aligned}\right.
$$

For the above closed-loop system, we can write:

$$
\begin{aligned}
& y(k+l)=h^{\prime}[x(k)], \quad l=0, \ldots, r-1 \\
& y(k+r)=h^{r-1}\left(\Phi \left(x(k), \Psi_{o}\left(x(k), d_{\mathbb{B}}(k),\right.\right.\right. \\
& \left.\left.\left.\left(1+\sum_{l=1}^{r} \beta_{l}\right) v(k)-\sum_{l=1}^{r} \beta_{i} h^{r-l}[x(k)]\right), d_{\mathbb{Q}}(k), d_{\mathbb{B}}(k)\right)\right)
\end{aligned}
$$

Using the definition of $\Psi_{o}$ (Eqs. 21 and 22), the righthand side of the last equation in $\mathrm{Eq}$. A7:

$$
\begin{array}{r}
h^{r-1}\left(\Phi \left(x(k), \Psi_{o}\left(x(k), d_{\Theta B}(k),\left(1+\sum_{l=1}^{r} \beta_{l}\right) v(k)\right.\right.\right. \\
\left.\left.\left.-\sum_{l=1}^{r} \beta_{l} h^{r-1}[x(k)]\right), d_{Q}(k), d_{\Theta}(k)\right)\right) \\
=\left(1+\sum_{l=1}^{r} \beta_{l}\right) v(k)-\sum_{l=1}^{r} \beta_{l} h^{r-1}[x(k)]
\end{array}
$$

therefore:

$$
y(k+r)=\left(1+\sum_{l=1}^{r} \beta_{l}\right) v(k)-\sum_{l=1}^{r} \beta_{l} h^{r-l}[x(k)]
$$

which is equivalent to:

$$
y(k+r)+\sum_{l=1}^{r} \beta_{1} y(k+r-l)=\left(1+\sum_{l=1}^{r} \beta_{l}\right) v(k)
$$

This closed-loop response is independent of the $Q$ - and $Q$-type disturbances; these disturbances are completely rejected by the feedforward/feedback law of Eq. 24.

Part $b$. Under the feedforward/feedback law of Eq. 24 with $\beta_{1}=\cdots=\beta_{r}=0$, the closed-loop system becomes:

$$
\begin{aligned}
x(k+1) & =\Phi\left\{x(k), \Psi_{o}\left[x(k), d_{\mathbb{B}}(k), d_{\mathbb{C}}(k), v(k)\right],\right. \\
& \left.d_{Q}(k), d_{\mathbb{B}}(k), d_{\mathbb{C}}(k)\right\} \\
y(k) & =h[x(k)]
\end{aligned}
$$

Consider the above closed-loop system and make a step change in the $\mathfrak{C}$-type disturbances at time instant $k$, keep $v$ unchanged $\left[v(k+l)=v_{o}, l=0,1, \ldots\right]$, and assume at time instant $k$ the closed-loop system is at steady-state, that is, $x(k+1)=x(k)$ and $y(k)=v_{0}$. For the above closed-loop system, we can write:

$$
\begin{aligned}
y(k) & =h[x(k)]=v_{o} \\
y(k+l) & =h^{\prime}\left[x(k), d_{\mathrm{e}}(k)\right] \quad l=1, \ldots, r-1 \\
y(k+r) & =h^{r-1}\left\{\Phi \left[x(k), \Psi_{o}\left[x(k), d_{\mathbb{B}}(k), d_{\mathrm{e}}(k), v_{o}\right],\right.\right. \\
& \left.\left.d_{\mathbb{Q}}(k), d_{\mathbb{B}}(k), d_{\mathrm{e}}(k)\right], d_{\mathfrak{C}}(k)\right\}
\end{aligned}
$$

Using the definition of $\Psi_{o}$ (Eqs. 21 and 22), the righthand side of the last equation in Eq. A8:

$$
\begin{aligned}
h^{r-1}\left\{\Phi \left[x(k), \Psi_{o}\left[x(k), d_{B}(k), d_{\mathrm{C}}(k), v_{o}\right],\right.\right. \\
\left.\left.d_{\mathbb{Q}}(k), d_{\mathbb{Q}}(k), d_{\mathrm{C}}(k)\right], d_{\mathrm{C}}(k)\right\}=v_{0}
\end{aligned}
$$

therefore, in the presence of the step changes in the e-type disturbances and constant $v$ the output will return to $v$ after $r$ sampling periods from the time of applying the changes, that is, ISE-optimal rejection of the e-type measurable disturbances. Q.E.D.

\section{Proof of Theorem 5}

The proof of this theorem involves application of Lyapunov's first method as follows. Since the system of Eq. 1 has hyperbolically stable finite zero dynamics, this means (by Definition 4) that all the eigenvalues of the matrix $\mathfrak{d}_{0}(0,0,0)$, where $J_{o}$ is given by Eq. 27, are in the interior of the unit circle. Using the definition of the relative order $r$ and the standard matrix identities:

$$
\begin{aligned}
& \operatorname{det}\left(z I-\mathfrak{d}_{0}(0,0,0)\right)=\operatorname{det}\left(z I-\frac{\partial \Phi(0,0,0)}{\partial x}\right) \\
&+\frac{\frac{\partial h(0)}{\partial x}\left[\frac{\partial \Phi(0,0,0)}{\partial x}\right]^{r}}{\frac{\partial h(0)}{\partial x}\left[\frac{\partial \Phi(0,0,0)}{\partial x}\right]^{r-1} \frac{\partial \Phi(0,0,0)}{\partial u}} \\
& \cdot \operatorname{adj}\left(z I-\frac{\partial \Phi(0,0,0)}{\partial x}\right) \frac{\partial \Phi(0,0,0)}{\partial u}
\end{aligned}
$$

and (see also Eq. 43)

$$
\begin{gathered}
z^{\prime} \frac{\partial h(0)}{\partial x} \operatorname{adj}\left(z I-\frac{\partial \Phi(0,0,0)}{\partial x}\right) \frac{\partial \Phi(0,0,0)}{\partial u} \\
=\frac{\partial h(0)}{\partial x}\left[\frac{\partial \Phi(0,0,0)}{\partial x}\right]^{r-1} \frac{\partial \Phi(0,0,0)}{\partial u} \cdot \operatorname{det}\left(z I-\frac{\partial \Phi(0,0,0)}{\partial x}\right) \\
+\frac{\partial h(0)}{\partial x}\left[\frac{\partial \Phi(0,0,0)}{\partial x}\right]^{r} \\
\cdot \operatorname{adj}\left(z I-\frac{\partial \Phi(0,0,0)}{\partial x}\right) \frac{\partial \Phi(0,0,0)}{\partial u}
\end{gathered}
$$


where $I$ is the $n \times n$ identity matrix, one finds the characteristic polynomial of the matrix $\mathcal{J}_{o}(0,0,0)$ to be:

$$
\frac{z^{r} \frac{\partial h(0)}{\partial x} \operatorname{adj}\left(z I-\frac{\partial \Phi(0,0,0)}{\partial x}\right) \frac{\partial \Phi(0,0,0)}{\partial u}}{\frac{\partial h(0)}{\partial x}\left[\frac{\partial \Phi(0,0,0)}{\partial x}\right]^{r-1} \frac{\partial \Phi(0,0,0)}{\partial u}}
$$

Therefore, the roots of the polynomial:

$$
\frac{\partial h(0)}{\partial x} \operatorname{adj}\left(z I-\frac{\partial \Phi(0,0,0)}{\partial x}\right) \frac{\partial \Phi(0,0,0)}{\partial u}
$$

all lie in the interior of the unit circle.

On the other hand, the local asymptotic stability characteristics of the closed-loop system of Theorem 5 are determined by the eigenvalues of the Jacobian matrix $\mathfrak{d}_{\beta}(0,0,0)$, given by:

$$
\begin{aligned}
\mathfrak{J}_{\beta}(x, u, d)= & {\left[\frac{\partial \Phi(x, u, d)}{\partial x}\right] } \\
& -\left[\frac{\partial \Phi(x, u, d)}{\partial u}\right]\left\{\frac{\partial}{\partial x} h^{r-1}[\Phi(x, u, d), d]\right. \\
& \left.+\sum_{l=1}^{r} \beta_{l} \frac{\partial h^{r-l}(x, d)}{\partial x}\right\}\left[\frac{\partial}{\partial u} h^{r-1}[\Phi(x, u, d), d]\right]^{-1}
\end{aligned}
$$

Using the definition of relative order $r$ and the standard matrix identities of Eqs. A9 and A10, one finds the characteristic polynomial of the matrix $\mathfrak{d}_{\beta}(0,0,0)$ to be:

$$
\left\{\begin{aligned}
\xi(k+1) & =A_{e} \xi(k)+b_{e}\left[y_{s p}(k)-y(k)\right] \\
\left(1+\sum_{l=1}^{r} \beta_{l}\right) v(k) & =c_{e} \xi(k)+f_{e}\left[y_{s p}(k)-y(k)\right]
\end{aligned}\right.
$$

and

$$
\begin{array}{r}
u(k)=\Psi_{o}\left(x(k), d_{\mathbb{B}}(k), d_{\mathcal{C}}(k),\left(1+\sum_{l=1}^{r} \beta_{l}\right) v(k)\right. \\
\left.-\sum_{l=1}^{r} \beta_{l} h^{r-l}\left[x(k), d_{\mathfrak{C}}(k)\right]\right)
\end{array}
$$

The subsystem of Eq. A12 is the realization of the external controller (Eq. 31), whose input/output behavior can be described by the difference operator representation:

$$
\begin{array}{r}
\frac{\left(q^{r}-1\right)+\gamma_{1}\left(q^{r-1}-1\right)+\cdots+\gamma_{r-1}(q-1)}{1+\gamma_{1}+\cdots+\gamma_{r}} v(k) \\
=\frac{\left(q^{r}+\beta_{1} q^{r-1}+\cdots+\beta_{r}\right)\left[y_{s p}(k)-y(k)\right]}{1+\beta_{1}+\cdots+\beta_{r}}
\end{array}
$$

where $q$ is the forward shift operator. On the other hand, the subsystem of Eq. A13 is the feedforward/feedback law of Eq. 24 , which induces the closed-loop response:

$$
\begin{aligned}
\left(q^{r}+\beta_{1} q^{r-1}+\cdots+\beta_{r-1} q+\beta_{r}\right) y(k) & \\
& =\left(1+\sum_{l=1}^{r} \beta_{l}\right) v(k)
\end{aligned}
$$

$$
\frac{\left(z^{r}+\beta_{1} z^{r-1}+\cdots+\beta_{r-1} z+\beta_{r}\right) \frac{\partial h(0)}{\partial x} \operatorname{adj}\left(z I-\frac{\partial \Phi(0,0,0)}{\partial x}\right) \frac{\partial \Phi(0,0,0)}{\partial u}}{\frac{\partial h(0)}{\partial x}\left[\frac{\partial \Phi(0,0,0)}{\partial x}\right]^{r-1} \frac{\partial \Phi(0,0,0)}{\partial u}}
$$

Because the roots of:

$$
z^{r}+\beta_{1} z^{r-1}+\cdots+\beta_{r-1} z+\beta_{r}=0
$$

are assumed to be all of the interior of the unit circle, it follows that all the eigenvalues of the matrix $d_{\beta}(0,0,0)$ will be in the interior of the unit circle and therefore the closed-loop system of Theorem 5 will be locally asymptotically stable. Q.E.D.

\section{Proof of Theorem 6}

Define the auxiliary variable:

$$
v(k)=\frac{c_{e} \xi(k)+d_{e}\left[y_{s p}(k)-y(k)\right]}{1+\sum_{l=1}^{r} \beta_{l}}
$$

Then Eq. 32 can be viewed as being composed of two subsystems: to the processes with $\rho_{j} \geq r, j=1, \ldots, p$. Combining Eqs. A14 and A15, we obtain the desirable closed-loop input/output dynamics:

$$
\begin{array}{r}
\left(q^{r}+\gamma_{1} q^{-1}+\cdots+\gamma_{r-1} q+\gamma_{r}\right) y(k) \\
=\left(1+\gamma_{1}+\cdots+\gamma_{r}\right) y_{s p}(k)
\end{array}
$$

that is, Eq. 30. Q.E.D.

\section{Proof of Theorem 7}

Using the auxiliary variable $v(k)$ defined by Eq. All, we see that the system of Eq. 35 is composed of two subsystems:

$$
\left\{\begin{aligned}
\xi(k+1) & =A_{e} \xi(k)+b_{e}\left[y_{s p}(k)-y(k)\right] \\
\left(1+\sum_{l=1}^{r} \beta_{l}\right) v(k) & =c_{e} \xi(k)+f_{e}\left[y_{s p}(k)-y(k)\right]
\end{aligned}\right.
$$




$$
\begin{array}{r}
w(k+1)=\Phi\left(w(k), \Psi_{\circ}\left(w(k), d_{\Theta}(k), d_{\mathrm{C}}(k),\right.\right. \\
\left.\left.\left(1+\sum_{i=1}^{r} \beta_{l}\right) v(k)-\sum_{i=1}^{r} \beta_{i} h^{r-1}\left[w(k), d_{\mathrm{e}}(k)\right]\right), d(k)\right) \\
u(k)=\Psi_{o}\left(w(k), d_{\Theta}(k), d_{\mathrm{C}}(k),\left(1+\sum_{i=1}^{r} \beta_{l}\right) v(k)\right. \\
\left.-\sum_{l=1}^{r} \beta_{i} h^{r-1}\left[w(k), d_{\mathrm{C}}(k)\right]\right) \quad(\mathrm{A} 17)
\end{array}
$$

The subsystem of Eq. A 16 is the realization of the external controller (Eq. 31), whose input/output behavior can be described by the difference operator representation:

$$
\begin{array}{r}
\frac{\left(q^{r}-1\right)+\gamma_{1}\left(q^{r-1}-1\right)+\cdots+\gamma_{r-1}(q-1)}{1+\gamma_{1}+\cdots+\gamma_{r}} v(k) \\
=\frac{\left(q^{r}+\beta_{1} q^{r-1}+\cdots+\beta_{r}\right)\left(y_{s p}(k)-y(k)\right)}{1+\beta_{1}+\cdots \cdot \beta_{r}}
\end{array}
$$

On the other hand, the subsystem of Eq. A17 consists of a feedforward/state feedback law, with the states being reconstructed via the open-loop observer of Eq. 33. Hence, under consistent initialization of $w$ and $x$ (that is, $w(0)=x(0)$ ), we will have $w(k)=x(k)$ for all $k$, and therefore, Eq. A17 induces the dynamics:

$$
\begin{aligned}
\left(q^{r}+\beta_{1} q^{-1}+\cdots+\beta_{r-1} q+\beta_{r}\right) y_{s p} & (k) \\
& =\left(1+\sum_{l=1}^{r} \beta_{l}\right) v(k)
\end{aligned}
$$

to the processes with $\rho_{j} \geq r, j=1, \ldots, p$. Combining Eqs. A18 and $A 19$, we obtain the desirable closed-loop input/output dynamics:

$$
\left(q^{r}+\gamma_{1} q^{r-1}+\cdots+\gamma_{r}\right) y(k)=\left(1+\gamma_{1}+\cdots+\gamma_{r}\right) y_{s p}(k)
$$

that is, Eq. 30. Q.E.D.

\section{Proof of Corollary 1}

At first observe that, from the space-state realization of the external controller (Eq. 31):

$$
\begin{aligned}
\left(1+\sum_{l=1}^{r} \beta_{l}\right) v(k) & =\xi_{l}(k+1)+\sum_{l=1}^{r} \beta_{l} \xi_{l}(k) \\
& =\xi_{r}(k+r)+\sum_{l=1}^{r} \beta_{l} \xi_{r}(k+r-l)
\end{aligned}
$$

On the other hand, from Eqs. 33 and 34, we see that:

$h[w(k+r)]+\sum_{l=1}^{r} \beta_{l} h(w(k+r-l))$

$$
=\left(1+\sum_{l=1}^{r} \beta_{l}\right) v(k)
$$

for processes with $\rho_{j} \geq r, j=1, \ldots, p$. Combining Eqs. A20 and $A 21$, we conclude that:

$$
\begin{aligned}
h[w(k+r)]+\sum_{l=1}^{r} \beta, h[w(k+r-l)] & \\
& =\xi_{r}(k+r)+\sum_{l=1}^{r} \beta_{l} \xi_{r}(k+r-l)
\end{aligned}
$$

Consequently, if the external controller and the observer are consistently initialized according to:

$$
\xi(0)=h^{r-l}[w(0)], \quad l=1, \ldots, r
$$

that is:

$$
\xi_{r}(r-l)=h[w(r-l)], \quad l=1, \ldots, r
$$

we will have $\xi_{r}(k)=h[w(k)], \forall k$, and therefore:

$$
\xi_{l}(k)=h^{r-l}[w(k)], \quad \forall k, l=1, \ldots, r
$$

Substituting this into the controller of Eq. 35, we obtain Eq. 36. Q.E.D.

Manuscript received Nov. 25, 1992, and revision received Jun. 17, 1993. 